\title{
Reduction of Truncation Errors in Planar, Cylindrical, and Partial Spherical Near-Field Antenna Measurements
}

\author{
Francisco José Cano-Fácila, ${ }^{1}$ Sergey Pivnenko, ${ }^{2}$ and Manuel Sierra-Castañer ${ }^{1}$ \\ ${ }^{1}$ Radiation Group, Signals, Systems and Radiocommunications Department, Technical University of Madrid, \\ Avenue Complutense 30, 28040 Madrid, Spain \\ ${ }^{2}$ Department of Electrical Engineering, Technical University of Denmark, 2800 Kongens, Lyngby, Denmark
}

Correspondence should be addressed to Francisco José Cano-Fácila, francisco@gr.ssr.upm.es

Received 12 July 2011; Accepted 8 September 2011

Academic Editor: Claudio Gennarelli

Copyright ( 2012 Francisco José Cano-Fácila et al. This is an open access article distributed under the Creative Commons Attribution License, which permits unrestricted use, distribution, and reproduction in any medium, provided the original work is properly cited.

A method to reduce truncation errors in near-field antenna measurements is presented. The method is based on the GerchbergPapoulis iterative algorithm used to extrapolate band-limited functions and it is able to extend the valid region of the calculated far-field pattern up to the whole forward hemisphere. The extension of the valid region is achieved by the iterative application of a transformation between two different domains. After each transformation, a filtering process that is based on known information at each domain is applied. The first domain is the spectral domain in which the plane wave spectrum (PWS) is reliable only within a known region. The second domain is the field distribution over the antenna under test (AUT) plane in which the desired field is assumed to be concentrated on the antenna aperture. The method can be applied to any scanning geometry, but in this paper, only the planar, cylindrical, and partial spherical near-field measurements are considered. Several simulation and measurement examples are presented to verify the effectiveness of the method.

\section{Introduction}

In many cases, antenna parameters, such as gain, directivity, radiation pattern, side-lobe level, and beamwidth, cannot be determined directly from measurements that are obtained in a far-field range because the distance to the far-field region may be too large. However, it is well known that those parameters can be obtained using analytical transformations from near-field measurements [1-4]. Moreover, these types of measurement can be performed in indoor ranges, reducing unwanted contributions from the environment, such as reflections or diffractions, as much as possible.

One important requirement to determine exact far-field patterns from near-field acquisitions is the ability to measure the electric or magnetic field that is tangential to an arbitrary surface that encloses the antenna under test (AUT). If that condition is satisfied, the field can be obtained anywhere outside the measurement surface and specifically in the farfield region by solving an integral over the surface on which the fields are known [1]. However, the solution of this integral is impractical to determine unless the scan surface supports orthogonal vector wave functions. There are only six coordinate systems that support vector wave functions that satisfy the orthogonality condition, but only three of them (planar, cylindrical, and spherical) are employed because of the simplicity of the required mechanical equipment. These three coordinate systems constitute the classical near-field antenna measurement systems and, although the three nearfield techniques are based on the same principle (measurement over a surface in the near-field and transformation to far-field), there are important differences among them. The planar and cylindrical scanning geometries are mechanically simpler than in the case of the spherical near-field (SNF). Moreover, the spherical near-field to far-field transformation is more complex, requiring more calculations to obtain the far-field pattern from the acquired data. However, the most accurate antenna patterns are obtained using this last type of acquisition because it is the only measurement setup where 
the AUT is fully enclosed by the acquisition surface. Therefore, there are no truncation errors in the calculated far-field pattern. In the planar near-field (PNF) and cylindrical nearfield (CNF) measurements, because of the finite size of the scan surface, the closed surface condition is never fulfilled, and, consequently, the true far-field pattern is never known in the whole sphere, that is, the pattern is only valid within the called reliable region. A second effect, which is caused by the discontinuity of the measured field at the edge of the scan surface, is the presence of a ripple within this region.

Because the truncation error is an unavoidable error in PNF and CNF measurements, and it is not present in SNF measurements, most of the approaches to reduce this kind of error have been specifically proposed for the two first configurations. These approaches can be divided into two groups. The first group attempts to reduce the second effect that was mentioned previously, that is, the ripple within the reliable region by the application of proper window functions to the near-field data. In $[5,6]$, a directive synthetic array of probes is created by combining near-field data points to reduce the level at the edges of the measurement plane. This approach provides noticeable ripple reduction, especially for the measurement of low-directive antennas. Another ripple reduction approach is proposed in $[7,8]$ in which raised cosine amplitude and quadratic phase windows are shown to provide high accuracy. Although windowing techniques can greatly reduce erroneous ripples, an extra scan area is needed to obtain the same reliable region. If the scan area is not extended with the use of one of these techniques, the extent of the reliable region is reduced.

The approaches of the second group do not attempt to reduce the ripple within the region in which the farfield pattern is reliable, but they attempt to obtain a good estimation of the true pattern outside of that region. One of these approaches, called the equivalent magnetic current approach [9], presents a method of computing far-fields in the whole forward hemisphere from planar near-field measurements. The idea is to compute the equivalent magnetic currents in the AUT plane by solving the system of integral equations that relate these currents and the measured field. Once these currents are known, it is possible to produce the correct far-fields in front of the AUT. The main drawback of this approach is the great computational complexity that is required to solve the system of integral equations. However, that complexity may be drastically reduced by using a magnetic dipole array approximation instead of the equivalent magnetic current approach, eliminating the numerical integration in the process. Another strategy to reduce truncation errors is to rotate the AUT about one or more axes [10], measuring in different planes and combining them to increase the maximum validity angle. Logically, this technique requires a particular near-field to far-field transformation for each combination of plane acquisitions. In [11], the problem of truncation is addressed using a priori information about the AUT. The main idea of this approach is to estimate the nearfield data outside of the scanning area by extrapolating the measured data before calculating the far-field pattern. The a priori information is employed to obtain a nonredundant and nonuniform representation [12] of the samples that are taken over the measurement surface. Thanks to this distribution of the samples, a large amount of samples outside of the scan plane can be recovered with good accuracy. This strategy was experimentally validated in [13], obtaining good far-field results for the case of cylindrical scanning. The same idea of nonredundant and nonuniform representation of electromagnetic fields was also used in [14] for extrapolating the data outside of a plane-polar scanning. In this last work, however, optimal sampling interpolation expansions instead of the cardinal series expansions employed in $[11,12]$ are applied. In [14], it is numerically demonstrated that these new expansions guarantee a better reconstruction of the samples outside the measurement surface for a given number of measurement points. However, these extrapolation methods are not able to remove the truncation error in all the far-field pattern. A new method that is based on the same principle, that is, the use of a nonredundant sampling in the acquisition, was proposed in [15]. In this method, the truncation error is practically eliminated by also addressing points in surfaces external to the actual scanning area. Therefore, this method can be applied whenever the set-up allows the variation of the distance between the AUT and the probe. Another alternative to increase the reliable region by extrapolating the planar near-field data is described in [16]. The extrapolation is achieved by first back-propagating the measured field to the AUT plane. After that, only the samples within the AUT aperture are retained, and they are used to restore the external samples in terms of a diffraction integral along the aperture rim. Finally, the field is transformed back to the measurement plane to obtain new field samples outside of the measurement region. The main drawback of this last approach is, as in $[11,12,14]$, the impossibility of recovering samples over an infinite surface with good accuracy, and, therefore, the truncation error is not completely removed for large elevation angles. A recent publication [17] also uses $a$ priori information about the AUT in an iterative algorithm to extrapolate the reliable portion of the calculated far-field pattern. This method was proposed for the planar near-field case, and the theoretical basis of the iterative algorithm was presented in $[18,19]$ by Gerchberg and Papoulis, respectively.

As commented before, truncation errors are always present in PNF and CNF measurements but not in SNF measurements. However, there are special cases, for example, when measuring electrically large antennas, in which the measurement time may be prohibitively long and an acquisition over the whole sphere is not practical. A solution to reduce the data acquisition time is to measure over a partial sphere. Nevertheless, the new acquisition surface does not fully enclose the AUT, and, as in the PNF and CNF cases, a truncation error appears in the far-field pattern. Although this is a typical error in PNF and CNF measurements, there are also some studies that have dealt with this problem in partial SNF measurements. In [20], the truncated spherical near-field data are used to calculate an equivalent currents distribution of a set of dipoles that reproduce the radiation of the AUT. As in [9], once that distribution is known, the farfield pattern is easily computed in the entire sphere. When measuring over a partial sphere, the error that appears in the computed far-field arises from the nonorthogonality of 
the classical spherical modal functions in the uncompleted surface. Therefore, the calculated modal coefficients are incorrect. This problem can be solved by employing a new basis function set that is orthogonal over the truncated angular domain. It is subsequently necessary to derive a nearfield to far-field transformation for the resulting coefficients. This solution was presented for two-dimensional cylindrical/spherical near-field scanning in [21] and for threedimensional acoustic spherical near-field scanning in [22]. The alternative expansion considered in these works is based on Slepian functions [23]. In some cases, it is not the measurement time that is the limitation of SNF measurements, but the impossibility to get reliable data over the whole sphere, for example, due to support-structure blockage when measuring electrically small antennas. In [24], a method that is based on a least-squares technique is employed to calculate the spherical wave coefficients using forward hemisphere data only. This method can significantly reduce truncation errors, but it is not efficient for large antennas. This problem is also addressed in [25] where the band-limited property of the spherical wave coefficients is exploited in an iterative algorithm that substitutes the unreliable portion of the measurement sphere with new samples at each iteration.

In the present work, a method to reduce truncation errors when measuring the field overtruncated surfaces is developed. The method is based on the iterative algorithm that was proposed in $[18,19]$, and it has already been applied to the PNF case in [17]. However, this method can be applied to any scanning geometry by taking certain considerations into account. Therefore, this work can be viewed as a generalization of the method presented in [17]. Moreover, compared to some aforementioned approaches, it is not necessary to take samples in surfaces external to the actual scanning area. Therefore the reduction of truncation errors is achieved without increasing the measurement time. In addition, the computational cost is not large because the method is based on the iterative application of Fourier transforms between the plane wave spectrum (PWS) and the extreme near-field, and those transforms can be made quite rapid by using fast fourier transform techniques, not as in [9] where the equivalent magnetic currents are obtained by solving a complex system of integral equations.

A bottleneck of the method presented in [17] may be the time required to find the optimum termination point in the iterative procedure. In this work, a faster procedure based on the Gradient Descent algorithm, with which is possible to obtain the iteration number where the error is minimum, is proposed.

The method provides an exact reconstruction when starting from error free data. In practice, the measured data are always affected by errors, and therefore, the method does not converge to the exact solution. However, the method is very insensitive to errors and is able to provide a very good radiation pattern reconstruction using initial data corrupted by noise or other measurement errors.

The main limitation of the method is that the truncation error can only be removed in the forward hemisphere. Moreover, it is within the methods that require a priori information about the AUT, like all the methods based on a non- redundant and nonuniform representation of the samples [11-15]. In our case, it is necessary to know exactly the AUT dimensions. Due to this fact, the best results are obtained when the AUT is an aperture antenna because its dimensions are well defined.

Although our method is a generic approach that can be applied to any scanning geometry, only the most common truncated cases (plane, cylinder, and partial sphere) are considered here.

The paper is organized as follows. Section 2 gives an overview of the concept of the spectral reliable region. The method to reduce truncation errors is described in Section 3 where its convergence is also studied. Section 4 presents a simulated model that is used in Section 5 to analyze the critical aspects of the method. The effectiveness of the method is validated in Section 6 using both the simulated data from Section 4 and measured near-field data. Conclusions are drawn in Section 7.

\section{Spectral Reliable Region}

The classical way for obtaining the far-field pattern from near-field measurements is to employ the modal expansion method, which is based on the fact that the field over an appropriate surface can be expressed as a linear combination of a set of orthogonal functions. In this method, after the measurement of the tangential components of the electric or magnetic field over a truncated surface, the modal coefficients are obtained by making use of the orthogonalities of the modal functions. However, these functions are orthogonal over the original surface but not over the truncated surface, so these coefficients are calculated erroneously. This error is translated into two different effects in the calculated far-field pattern. On one hand, the results are completely unreliable outside of a certain spectral region. On the other hand, erroneous ripples appear within that region due to the discontinuity of the measured field at the edge of the truncated surface. Therefore, the entire calculated pattern is always affected by errors, and it is not possible to define a region where the error is completely zero. However, the concept of the spectral reliable region is usually applied to refer to the region in which the error is not negligible but is low. A classical definition of this region is given in $[3,26]$, where geometrical optics is employed to calculate the maximum validity angles that are used to define the reliable region. According to this definition, one particular direction will be within the reliable region when the scan surface includes all of the rays that are parallel to the direction coming from any part of the AUT as shown in Figure 1(a). The opposite situation is depicted in Figure 1(b) in which there are rays that do not lie on the scan surface.

The reliable region defined in this way depends on the AUT size, the distance from the scan surface to the AUT and the shape and size of the acquisition surface. Therefore, even with the use of the same AUT and the same measurement distance, the reliable region will be different for each type of measurement setup and require an independent analysis for each of them. As mentioned before, not only the classical 


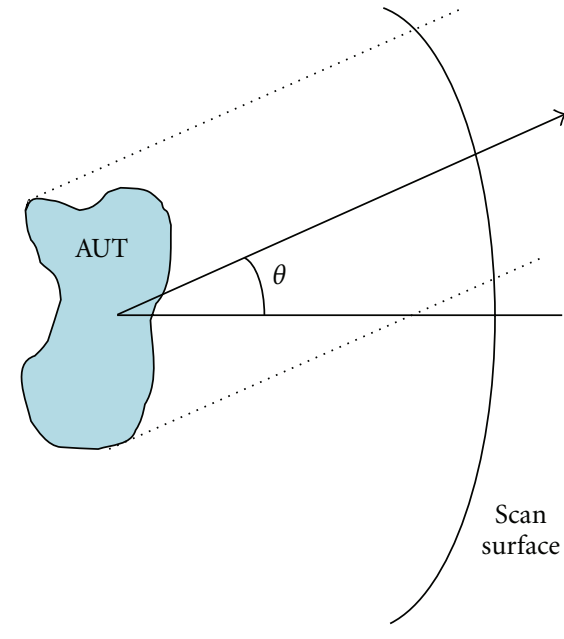

(a)

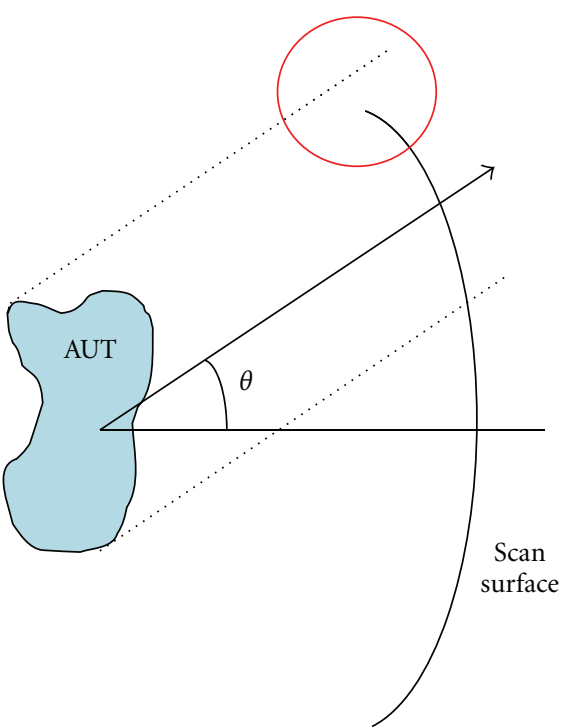

(b)

FIgURE 1: Geometrical optics employed to define the spectral reliable region: (a) direction included in the reliable region; (b) direction not included in the reliable region.

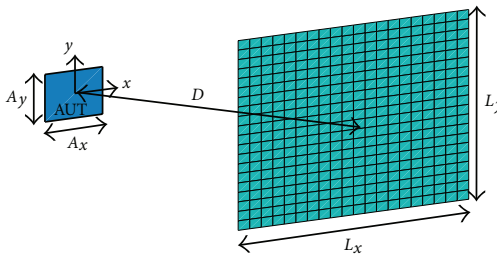

(a)

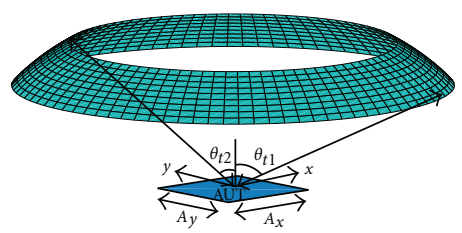

(d)

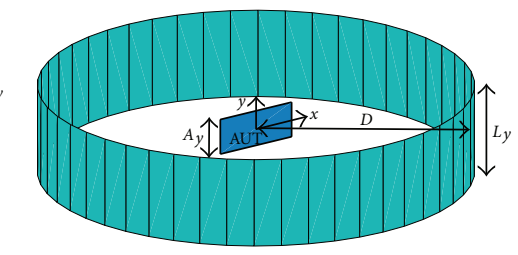

(b)

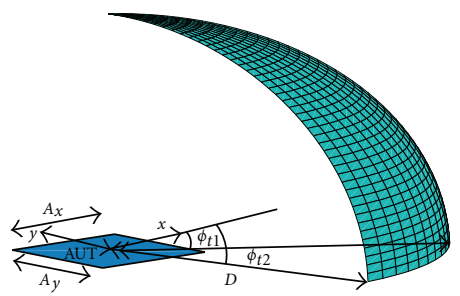

(e)

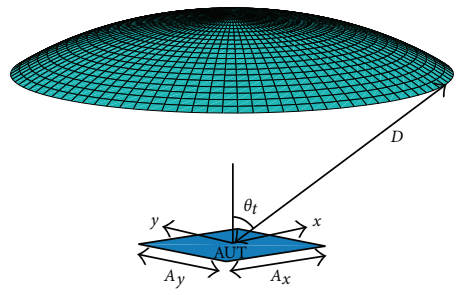

(c)

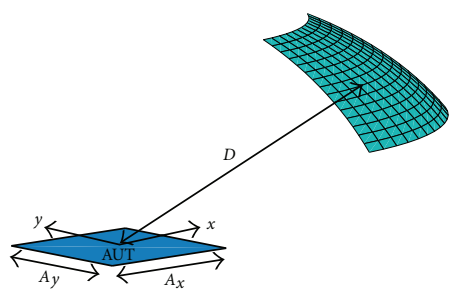

(f)

FIgure 2: Truncation in near-field measurement setups: (a) Planar truncation; (b) Cylindrical truncation; (c) Polar truncation; (d) Spherical ring truncation; (e) Azimuthal truncation; (f) Sectorial truncation.

cases of truncation, that is, the PNF and CNF cases, but also the partial SNF measurements are analyzed here. Logically, because it is possible to sample over different parts of the sphere, there are different types of reliable region, not as in the two first cases, where the shape of the reliable region is always the same. The choice of the sampling region will depend on the expected radiation pattern because if most of the radiated energy is concentrated in that region, the proposed method will provide a better reconstruction. In particular, besides the planar and cylindrical truncation, four different truncations in SNF measurements are studied in this work. The six truncation surfaces for different measurement setups are depicted in Figure 2, and their cor- responding reliable regions are shown in Figure 3. These reliable regions were determined using geometrical optics, and their mathematical formulations are indicated in Table 1.

\section{Extrapolation Method}

As shown in the previous section, when measuring over a truncated surface, the calculated far-field pattern can only be considered reliable within a limited known region. Therefore, the truncation problem can be viewed as an extrapolation problem. Various methods have been presented to achieve satisfactory estimates when extrapolating a known portion of a signal. Most of these methods are general 


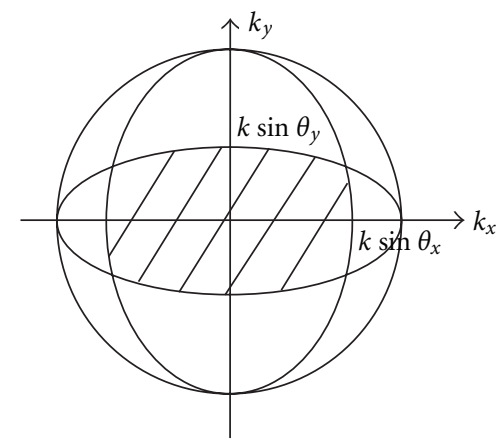

(a)

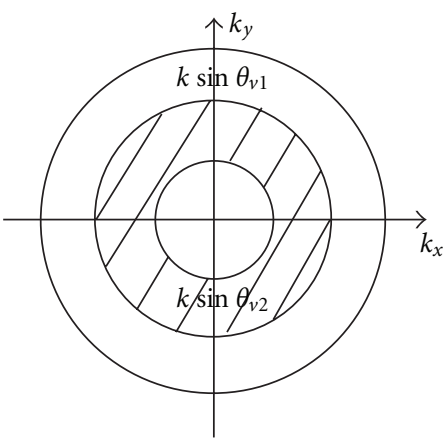

(d)

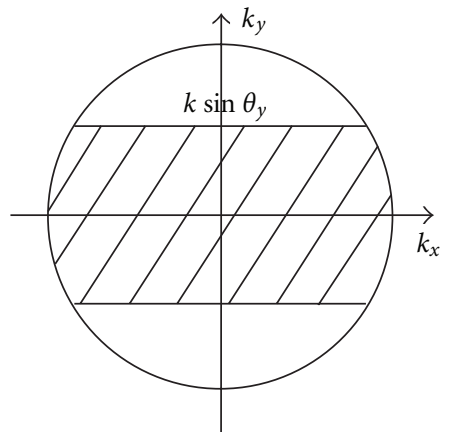

(b)

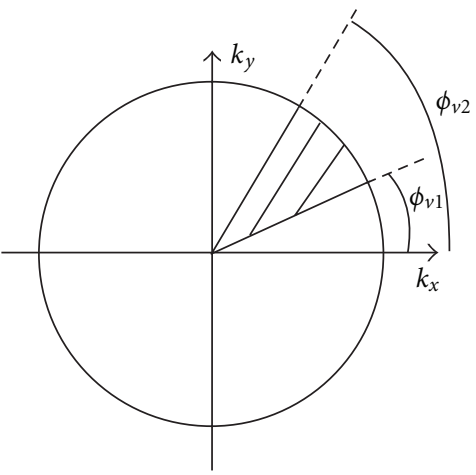

(e)

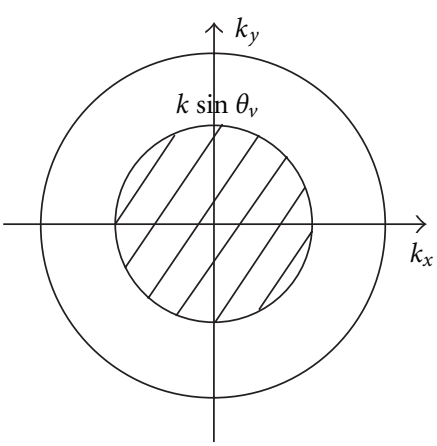

(c)

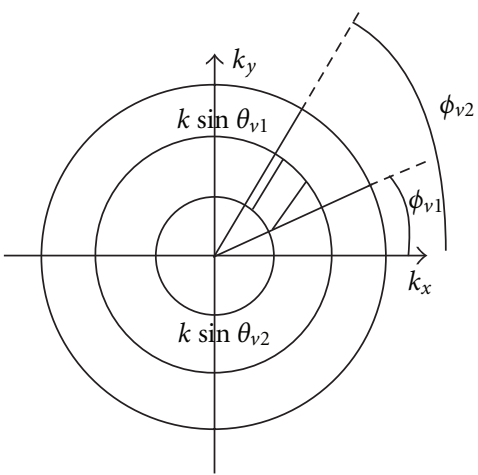

(f)

FIGURE 3: Spectral reliable regions: (a) PNF measurement; (b) CNF measurement; (c) SNF measurement with polar truncation; (d) SNF measurement with spherical ring truncation; (e) SNF measurement with azimuthal truncation; (f) SNF measurement with sectorial truncation.

TABLE 1: Determination of the spectral reliable regions based on geometrical optics for each type of measurement setup.

\begin{tabular}{lll}
\hline Measurement set-up & Maximum validity angles & Spectral reliable region \\
\hline PNF measurement & $\begin{array}{l}\theta_{x}=\arctan \left(\left(L_{x}-A_{x}\right) / 2 D\right) ; \\
\theta_{y}=\arctan \left(\left(L_{y}-A_{y}\right) / 2 D\right)\end{array}$ & $\left\{k_{x}^{2} /\left(k \sin \theta_{x}\right)^{2}+k_{y}^{2} / k^{2}<1\right\} \cap\left\{k_{x}^{2} / k^{2}+k_{y}^{2} /\left(k \sin \theta_{y}\right)^{2}<1\right\}$ \\
\hline CNF measurement & $\theta_{y}=\arctan \left(\left(L_{y}-A_{y}\right) / 2 D\right)$ & $\left\{\left|k_{y}\right|<k \sin \theta_{y}\right\}$ \\
\hline $\begin{array}{l}\text { SNF measurement with } \\
\text { polar truncation }\end{array}$ & $\theta_{v}=\theta_{t}-\arcsin \left(r_{0} / D\right)^{*}$ & $\left\{k_{x}^{2}+k_{y}^{2}<\left(k \sin \theta_{v}\right)^{2}\right\}$ \\
\hline $\begin{array}{l}\text { SNF measurement with } \\
\text { spherical ring truncation }\end{array}$ & $\theta_{v 1}=\theta_{t 1}-\arcsin \left(r_{0} / D\right) ; \theta_{v 2}=\theta_{t 2}+\arcsin \left(r_{0} / D\right)^{*}$ & $\left\{k_{x}^{2}+k_{y}^{2}<\left(k \sin \theta_{v 1}\right)^{2}\right\} \cap\left\{k_{x}^{2}+k_{y}^{2}>\left(k \sin \theta_{v 2}\right)^{2}\right\}$ \\
\hline $\begin{array}{l}\text { SNF measurement with } \\
\text { azimuthal truncation }\end{array}$ & $\phi_{v 1}=\phi_{t 1}+\arcsin \left(r_{0} / D\right) ; \phi_{v 2}=\phi_{t 2}-\arcsin \left(r_{0} / D\right)^{*}$ & $\left\{k_{y}>k_{x} \cdot \tan \phi_{v 1}\right\} \cap\left\{k_{y}<k_{x} \cdot \tan \phi_{v 2}\right\}$ \\
\hline $\begin{array}{l}\text { SNF measurement with } \\
\text { sectorial truncation }\end{array}$ & $\begin{array}{l}\theta_{v 1}=\theta_{t 1}-\arcsin \left(r_{0} / D\right) ; \theta_{v 2}=\theta_{t 2}+\arcsin \left(r_{0} / D\right)^{*} \\
\phi_{v 1}=\phi_{t 1}+\arcsin \left(r_{0} / D\right) ; \phi_{v 2}=\phi_{t 2}-\arcsin \left(r_{0} / D\right)^{*}\end{array}$ & $\begin{array}{l}\left.\left\{k_{x}^{2}+k_{y}^{2}<\left(k \sin \theta_{v 1}\right)^{2}\right\} \cap\left\{k_{x}^{2}+k_{x} \cdot \tan \phi_{v 1}\right\} \cap\left\{k \sin \theta_{v 2}\right)^{2}\right\} \\
{ }^{*} r_{0}=(1 / 2) \sqrt{A_{x}^{2}+A_{y}^{2}} .\end{array}$
\end{tabular}

approaches for the extrapolation of the finite-time segment of a signal, and they are based on deconvolution or predictive filtering. However, there are special solutions that provide better results when the signal satisfies certain properties. This is the case in the alternating orthogonal projection method $[17,18]$, which consists of the sequential application of two signal operators in two different domains to obtain an approximation sequence with convergence to the desired extrapolation that is guaranteed in theory. This method is optimally designed for the extrapolation of the timetruncated version of a band-limited signal, that is, its Fourier transform is identically zero outside of some frequency set. Therefore, this last type of method can be employed in our truncation problem because it is necessary to extrapolate a truncated portion of the PWS with a Fourier transform (field distribution on the AUT plane) that is spatially band-limited because the field distribution is theoretically concentrated on the antenna aperture. 


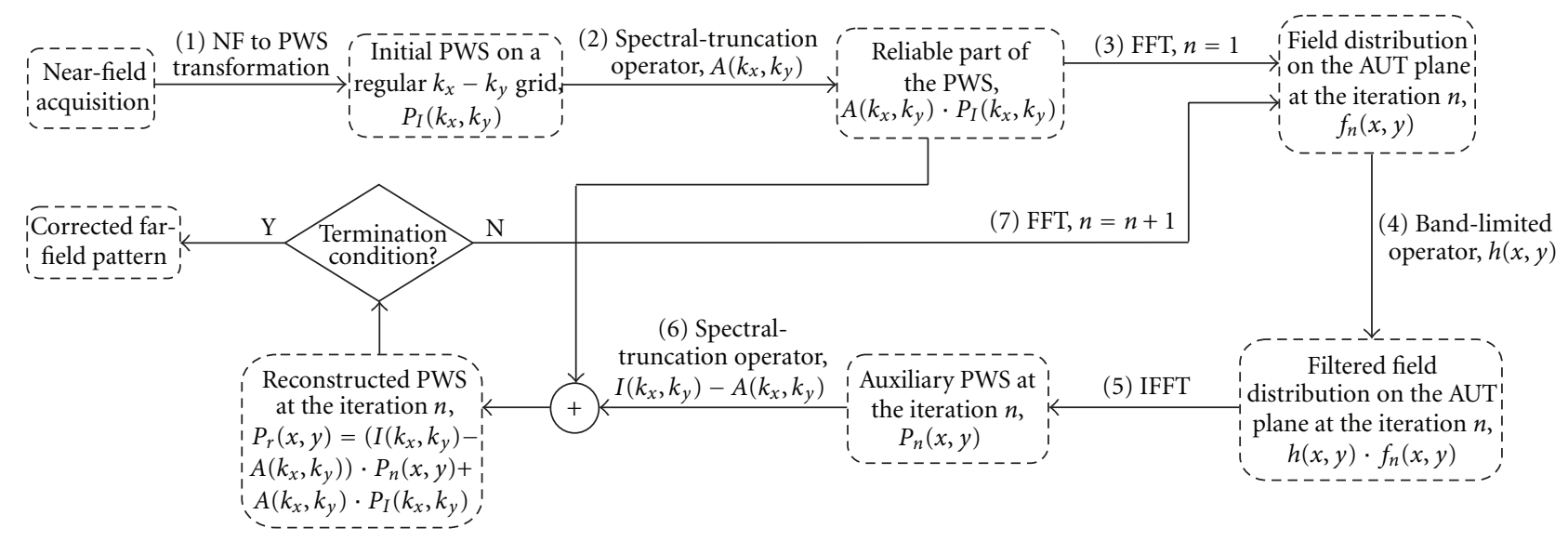

FIGURE 4: Schematic diagram of the method to reduce truncation errors. ${ }^{*} I\left(k_{x}, k_{y}\right)$ denotes the identity operator in the spectral domain.

Before describing all of the steps of the method, we present the two orthogonal projection operators that play an important role in the method. The first operator is applied in the spectral domain and defines the reliable portion of the PWS. This first operator is given by

$$
A\left(k_{x}, k_{y}\right)= \begin{cases}1, & \forall\left(k_{x}, k_{y}\right) \in \Omega_{R}, \\ 0, & \forall\left(k_{x}, k_{y}\right) \notin \Omega_{R},\end{cases}
$$

where $A$ is the spectral-truncation operator and $\Omega_{R}$ is one of the reliable regions that is defined in Table 1 . The second operator is called band-limited operator and is applied to the field distribution on the AUT plane:

$$
h(x, y)= \begin{cases}1, & \forall(x, y) \in \omega_{\mathrm{AUT}}, \\ 0, & \forall(x, y) \notin \omega_{\mathrm{AUT}},\end{cases}
$$

where $h$ stands for the band-limited operator and $\omega_{\mathrm{AUT}}$ is the region where the AUT is located. In the spectral domain, this last operator can be expressed as follows:

$$
B P\left(k_{x}, k_{y}\right)=P\left(k_{x}, k_{y}\right) * H\left(k_{x}, k_{y}\right)
$$

being $B$ the band-limited operator defined in the spectral domain, $P\left(k_{x}, k_{y}\right)$ represents the PWS, and $H$ is the inverse Fourier transform of the operator $h$.

The schematic diagram of the method is shown in Figure 4 and the steps are described as follows.

Step 1. Near-field data are used to calculate the PWS. Because the fast fourier transform algorithm is employed in the iterative part of the method, the samples of the PWS must be known on a regular $k_{x}-k_{y}$ grid.

Step 2. The unreliable portion of the initial PWS is filtered out by the application of the operator that was defined in (1).

Step 3. The field distribution over the AUT plane is obtained by taking the Fourier transform of the filtered PWS.
Step 4. The previous field distribution is spatially filtered using the band-limited operator that was presented in (2).

Step 5. The filtered field distribution is Fourier-transformed back to the spectral domain to obtain an auxiliary PWS.

Step 6. A new reconstructed PWS is calculated by substituting the unreliable portion of the initial PWS for the same portion of the PWS that was obtained in the previous step.

Step 7. If the new PWS fulfills the termination condition, the algorithm stops. If not, a new iteration starting from the Step 4 is performed.

3.1. Convergence. Once the method has been presented, its convergence is analyzed, that is, it is necessary to determine whether the method completely removes the truncation error and provides the exact solution outside of the reliable region. As deduced from Figure 4 , if the auxiliary PWS converges to the ideal PWS, we are ensuring the convergence of the method because the information of this PWS is employed to complete the unreliable region. The auxiliary PWS can be written at each iteration as follows

$$
\begin{gathered}
P_{1}=B A P_{I} \\
P_{2}=B[I-A] P_{1}+B A P_{I}=[I-B A] P_{1}+B A P_{I} \\
\vdots \\
P_{n}=B[I-A] P_{n-1}+B A P_{I}=[I-B A] P_{n-1}+B A P_{I}
\end{gathered}
$$

as a consequence, at the $n$th iteration, the error is given by

$$
P_{n}-P=[I-B A] P_{n-1}+B A P_{I}-P,
$$

where $P$ is the ideal PWS.

If the initial PWS calculated from near-field data does not contain errors within the reliable region, that is, $A P_{I}=A P$, expression (5) can be rewritten as

$$
P_{n}-P=[I-B A]\left(P_{n-1}-P\right)
$$


because $P_{n-1}$ and $P$ are band-limited functions, that is, $B P_{n-1}=P_{n-1}$ and $B P=P,(6)$ may be equivalently expressed as

$$
P_{n}-P=B[I-A]\left(P_{n-1}-P\right) .
$$

As observed from (7), the energy in the $n$th error PWS, as measured by the standard inner product $\varepsilon_{n}=\left\langle P_{n}-P, P_{n}-P\right\rangle$, is always less than or equal to the energy in the $(n-1)$ st error PWS. This reduction occurs because the operators $I-A$ and $B$ decrease the energy of the signal upon which they operate. Therefore, at each iteration, the error energy is reduced twice, as demonstrated as follows.

The energy in the $(n-1)$ st error PWS is

$$
\varepsilon_{n-1}=\left\langle P_{n-1}-P, P_{n-1}-P\right\rangle=\iint_{-\infty}^{\infty}\left|P_{n-1}-P\right|^{2} d k_{x} d k_{y}
$$

after applying the operator $I-A$, the new error energy is equal to

$$
\begin{aligned}
& \mathcal{E}_{n-1 / 2} \\
& \quad=\left\langle[I-A]\left(P_{n-1}-P\right),[I-A]\left(P_{n-1}-P\right)\right\rangle \\
& \quad=\iint_{-\infty}^{\infty}\left|P_{n-1}-P\right|^{2} d k_{x} d k_{y}-\iint_{\Omega_{R}}\left|P_{n-1}-P\right|^{2} d k_{x} d k_{y} \\
& \quad \leq \varepsilon_{n-1},
\end{aligned}
$$

using Parseval's identity, one can write

$$
\varepsilon_{n-1 / 2}=\frac{1}{2 \pi} \iint_{-\infty}^{\infty}|f|^{2} d x d y
$$

where $f$ is the Fourier transform of $[I-A]\left(P_{n-1}-P\right)$. Finally, the error energy at the $n$th iteration is given by

$$
\varepsilon_{n}=\langle h f, h f\rangle=\frac{1}{2 \pi} \iint_{\omega_{\mathrm{AUT}}}|f|^{2} d x d y \leq \varepsilon_{n-1 / 2} .
$$

Therefore, we have demonstrated that $\varepsilon_{n} \leq \varepsilon_{n-1 / 2} \leq \varepsilon_{n-1}$ for all $n$, ensuring that when starting from error free data in the reliable region, the method converges monotonically to the correct solution.

In the opposite case, that is, when the reliable portion of the PWS is affected by errors, we cannot write the expression (6) because $A P_{I} \neq A P$. However, the following relationship can be employed:

$$
\delta=A P-A P_{I}
$$

where $\delta$ is the difference between the calculated PWS and the ideal PWS within the reliable region. Therefore, expression (5) takes the form

$$
\begin{aligned}
P_{n}-P & =[I-B A]\left(P_{n-1}-P\right)-B \delta \\
& =B\left([I-A]\left(P_{n-1}-P\right)-\delta\right),
\end{aligned}
$$

in this case, the band-limited operator, $B$, also introduces an error reduction. Nevertheless, the error after the application of the first operator is

$$
\begin{aligned}
\varepsilon_{n-1 / 2}= & \left\langle[I-A]\left(P_{n-1}-P\right)-\delta,[I-A]\left(P_{n-1}-P\right)-\delta\right\rangle \\
= & \iint_{-\infty}^{\infty}\left|P_{n-1}-P\right|^{2} d k_{x} d k_{y}-\underbrace{\iint_{\Omega_{R}}\left|P_{n-1}-P\right|^{2} d k_{x} d k_{y}}_{I 1} \\
& +\underbrace{\iint_{\Omega_{R}}|\delta|^{2} d k_{x} d k_{y}}_{I 2},
\end{aligned}
$$

now, we can only ensure that $\varepsilon_{n-1 / 2} \leq \varepsilon_{n-1}$ if $I_{1} \leq I_{2}$, that is, when the difference between the auxiliary PWS in the $(n-1)$ st iteration and the ideal PWS is larger than the difference between the initial PWS and the ideal PWS within the reliable region. As will be shown later, this condition is satisfied in the initial iterations but not for large values of $n$ in which the difference between the auxiliary PWS and the ideal PWS is small and the initial error, $\delta$, is already dominant. Therefore, due to that initial error in the reliable portion of the PWS, the error in the estimated pattern initially decreases with the iteration number, but, after a certain number of iterations, the error starts to increase. The goal is to find the proper termination point in the iterative method.

\section{Simulated Models}

In the following sections, several results will be presented in order to analyze and validate the proposed method. The input data used to obtain these results are simulated nearfield data. The AUT that is employed in the simulations is an aperture with a Gaussian-tapered field distribution, as (15) shows:

$$
\vec{E}_{G}=E_{0} e^{-\left(x^{2} / 2 \sigma_{x}^{2}+y^{2} / 2 \sigma_{y}^{2}\right)} \hat{y}, \quad \vec{H}_{G}=\frac{1}{\eta} \hat{z} \times \vec{E}_{G} .
$$

The frequency is $12 \mathrm{GHz}$, and the simulations are carried out in the three measurement setups under study. The measurement distance is $100 \lambda$ for the three cases, and the sampling spacing and the size of the acquisition surfaces are indicated in Table 2 in which the type of phase excitation and the aperture size are also presented. The objective is to generate radiation patterns that steer in different directions with different beamwidths, especially to validate all of the truncation cases that are considered in partial SNF measurements. As observed from Table 2, Models I, II, and III are the same AUT but are measured in different scan surfaces. Models IV and $\mathrm{V}$ also have the same radiation pattern, but different acquisitions are employed in each of them. Finally, Model VI is measured using only one acquisition surface.

\section{Critical Aspects of the Method}

All steps of the proposed method are indicated in Section 3, however, some of these steps need to be described in more 
TABLE 2: Parameters of the simulated models.

\begin{tabular}{|c|c|c|c|c|c|c|c|}
\hline Model & $\begin{array}{l}\text { Measurement } \\
\text { setup }\end{array}$ & $\begin{array}{l}\text { Sampling } \\
\text { spacing }\end{array}$ & Measurement interval & Aperture size & Phase excitation & $\begin{array}{l}\text { Main beam } \\
\text { direction }\end{array}$ & $\begin{array}{l}\text { Radiation } \\
\text { pattern }\end{array}$ \\
\hline \multirow{2}{*}{ I } & \multirow{2}{*}{$\begin{array}{l}\text { PNF } \\
\text { measurement }\end{array}$} & $\Delta x=0.5 \lambda$ & $x=-0.9 \cdots 0.9 \mathrm{~m}$ & & & & \\
\hline & & $\Delta y=0.5 \lambda$ & $y=-0.9 \cdots 0.9 \mathrm{~m}$ & & & & \\
\hline \multirow{2}{*}{ II } & \multirow{2}{*}{$\begin{array}{l}\mathrm{CNF} \\
\text { measurement }\end{array}$} & $\Delta z=0.5 \lambda$ & $z=-1.25 \cdots 1.25 \mathrm{~m}$ & & Uniform & $\theta=0^{\circ}$ & \\
\hline & & $\Delta \phi=2^{\circ}$ & $\phi=0^{\circ} \cdots 359^{\circ}$ & $8 \lambda \times 8 \lambda$ & & & \\
\hline III & $\begin{array}{l}\text { SNF } \\
\text { measurement } \\
\text { with polar } \\
\text { truncation }\end{array}$ & $\begin{array}{l}\Delta \theta=2^{\circ} \\
\Delta \phi=2^{\circ}\end{array}$ & $\begin{array}{l}\theta=0^{\circ} \cdots 30^{\circ} \\
\phi=0^{\circ} \cdots 359^{\circ}\end{array}$ & & & & \\
\hline IV & $\begin{array}{l}\text { SNF } \\
\text { measurement } \\
\text { with spherical } \\
\text { ring truncation }\end{array}$ & $\begin{array}{l}\Delta \theta=2^{\circ} \\
\Delta \phi=2^{\circ}\end{array}$ & $\begin{array}{l}\theta=20^{\circ} \cdots 50^{\circ} \\
\phi=0^{\circ} \cdots 359^{\circ}\end{array}$ & $8 \lambda \times 8 \lambda$ & Progressive & $\begin{array}{l}\theta=35^{\circ} \\
\phi=180^{\circ}\end{array}$ & \\
\hline $\mathrm{V}$ & $\begin{array}{l}\text { SNF } \\
\text { measurement } \\
\text { with sectorial } \\
\text { truncation }\end{array}$ & $\begin{array}{l}\Delta \theta=2^{\circ} \\
\Delta \phi=2^{\circ}\end{array}$ & $\begin{array}{l}\theta=20^{\circ} \cdots 50^{\circ} \\
\phi=150^{\circ} \cdots 210^{\circ}\end{array}$ & & & & \\
\hline VI & $\begin{array}{l}\text { SNF } \\
\text { measurement } \\
\text { with azimuthal } \\
\text { truncation }\end{array}$ & $\begin{array}{l}\Delta \theta=2^{\circ} \\
\Delta \phi=2^{\circ}\end{array}$ & $\begin{array}{l}\theta=0^{\circ} \cdots 90^{\circ} \\
\phi=130^{\circ} \cdots 230^{\circ}\end{array}$ & $4 \lambda \times 16 \lambda$ & Progressive & $\begin{array}{l}\theta=30^{\circ} \\
\phi=180^{\circ}\end{array}$ & \\
\hline
\end{tabular}

detail. First, it is necessary to explain how to obtain the PWS on a regular grid in the spectral domain from nearfield data that are obtained in the three measurement setups. Second, a modified definition of the spectral reliable region is presented. Finally, an efficient algorithm to find the optimum termination point in the iterative method is proposed.

5.1. PWS on a Regular $k_{x}-k_{y}$ Grid. As observed in the description of the method, the fast fourier transform algorithm is used to calculate the field distribution over the AUT plane from the PWS and vice versa. This algorithm is computationally very efficient, but it only works with samples that are distributed on a regular grid in both domains, that is, the samples of the extreme near-field are obtained on a regular $x-y$ grid, and the PWS must be known on a regular $k_{x}-k_{y}$ grid.

In the PNF case, the PWS is directly obtained in the required grid because it is calculated as an inverse fast fourier transform of the measured samples taken over a regular $x-y$ grid.

In CNF and SNF cases, additional steps are required because the classical near-field to far-field transformations produce the final results on a regular $\theta-\phi$ grid. Different calculation approaches can be used, but the easiest one is to employ an interpolation algorithm to obtain the samples on the desired grid. This solution introduces an interpolation error, but it does not require a great computational cost.

Two other approaches provide the exact values on the desired grid, but they are more complex. Both of them use the information that is contained in the spherical wave coefficients (SWC), which are known in the SNF case, but not in the CNF case. However, they can be easily obtained from the far-field pattern [3].

One of these two approaches employs the sphericalwave-expansion-to-plane-wave-expansion (SWE-to-PWE) transformation that is presented in [27] in which it is demonstrated that it is possible to define a rigorous transformation to derive the PWS from the SWC,

$$
\begin{aligned}
P\left(k_{x}, k_{y}, z\right)= & \sum_{n=1}^{\infty} \sum_{m=-n}^{n} Q_{1 m n}^{(3)} T_{1 m n}\left(k_{x}, k_{y}, z\right) \\
& +Q_{2 m n}^{(3)} T_{2 m n}\left(k_{x}, k_{y}, z\right),
\end{aligned}
$$

where $Q_{1 m n}^{(3)}$ and $Q_{2 m n}^{(3)}$ are the outgoing SWC, and $T_{1 m n}$ and $T_{2 m n}$ are functions whose details can be found in [27].

The other approach evaluates the far-field pattern functions [3] on the desired directions that are defined by the regular $k_{x}-k_{y}$ grid and calculates the far-field pattern as follows:

$$
E_{\mathrm{FF}}(\theta, \phi)=L \sum_{s m n} Q_{s m n}^{(3)} K_{s m n}(\theta, \phi)
$$

where $E_{\mathrm{FF}}$ is the far-field pattern, $L$ is a constant, and $K$ are the far-field pattern functions. Logically, when the far-field pattern is known, the PWS can be easily obtained by solving a system of two linear equations.

5.2. Spectral Reliable Region Employed in the Method. As deduced from the convergence analysis of Section 3, when starting from exact data within the spectral reliable region, the proposed method converges to the correct solution. Otherwise, the error decreases with the iteration number, 


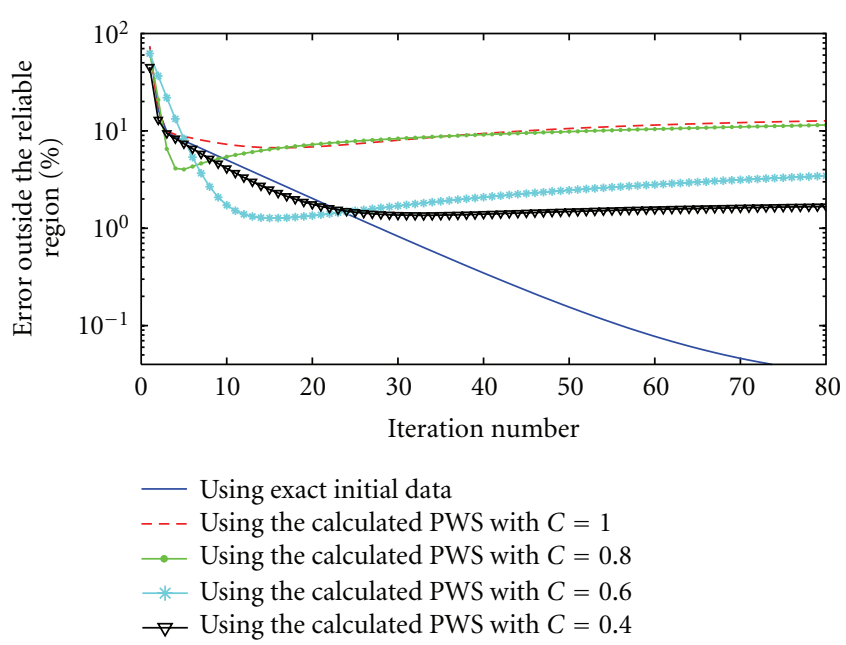

FIGURE 5: Error as a function of the iteration number using different reliable regions.

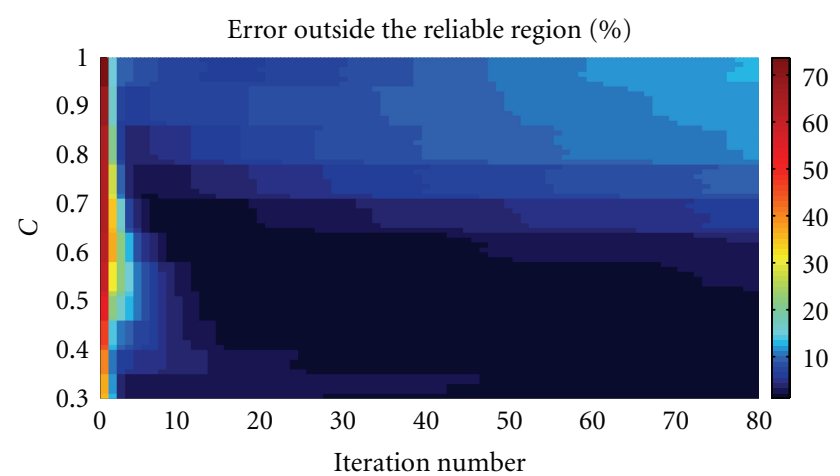

FIgURE 6: Error as a function of the iteration number and the parameter $A$.

but, after several iterations, it starts to increase. In Section 2, the classical definition of the spectral reliable region based on geometrical optics was presented. However, as noted in Section 1 , it is impossible to define a spectral region in which the error in the calculated far-field pattern is completely zero because of the unavoidable presence of ripple errors. Therefore, the error does not decrease monotonically in the iterative method. This effect can be observed in Figure 5, where the error variation with the iteration number is represented for the Model I using different spectral reliable regions. The error in this figure is calculated as

$$
\varepsilon_{n}(\%)=\frac{\sum_{i \notin \Omega_{R}}\left|E_{n}\left(\theta_{i}, \phi_{i}\right)-E_{R}\left(\theta_{i}, \phi_{i}\right)\right|^{2}}{\sum_{i \notin \Omega_{R}}\left|E_{R}\left(\theta_{i}, \phi_{i}\right)\right|^{2}} \cdot 100,
$$

where $E_{n}\left(\theta_{i}, \phi_{i}\right)$ and $E_{R}\left(\theta_{i}, \phi_{i}\right)$ are the electric field in the $n$th iteration and the reference electric field, respectively. As deduced from (18), only samples that are located outside of the reliable region are considered in the determination of the error.

Several conclusions can be extracted from Figure 5. As expected, the error decreases monotonically when using the
TABLE 3: Redefinition of the maximum validity angles.

\begin{tabular}{lc}
\hline Measurement setup & New maximum validity angles \\
\hline PNF measurement & $\theta_{x}^{\prime}=C \cdot \theta_{x} ; \theta_{y}^{\prime}=C \cdot \theta_{y}$ \\
\hline CNF measurement & $\theta_{y}^{\prime}=C \cdot \theta_{y}$ \\
\hline $\begin{array}{l}\text { SNF measurement with } \\
\text { polar truncation }\end{array}$ & $\theta_{v}^{\prime}=C \cdot \theta_{v}$ \\
\hline $\begin{array}{l}\text { SNF measurement with } \\
\text { spherical ring truncation }\end{array}$ & $\theta_{v 1}^{\prime}=\theta_{v 1}-(1-C) \cdot\left(\theta_{v 1}-\theta_{v 2}\right) / 2$ \\
SNF measurement with & $\theta_{v 2}^{\prime}=\theta_{v 2}+(1-C) \cdot\left(\theta_{v 1}-\theta_{v 2}\right) / 2$ \\
azimuthal truncation & $\phi_{v 1}^{\prime}=\phi_{v 1}+(1-C) \cdot\left(\phi_{v 1}-\phi_{v 2}\right) / 2$ \\
\hline & $\phi_{v 2}^{\prime}=\phi_{v 2}-(1-C) \cdot\left(\phi_{v 1}-\phi_{v 2}\right) / 2$ \\
SNF measurement with & $\theta_{v 1}^{\prime}=\theta_{v 1}-(1-C) \cdot\left(\theta_{v 1}-\theta_{v 2}\right) / 2$ \\
sectorial truncation & $\theta_{v 2}^{\prime}=\theta_{v 2}+(1-C) \cdot\left(\theta_{v 1}-\theta_{v 2}\right) / 2$ \\
& $\phi_{v 1}^{\prime}=\phi_{v 1}+(1-C) \cdot\left(\phi_{v 1}-\phi_{v 2}\right) / 2$ \\
& $\phi_{v 2}^{\prime}=\phi_{v 2}-(1-C) \cdot\left(\phi_{v 1}-\phi_{v 2}\right) / 2$ \\
\hline
\end{tabular}

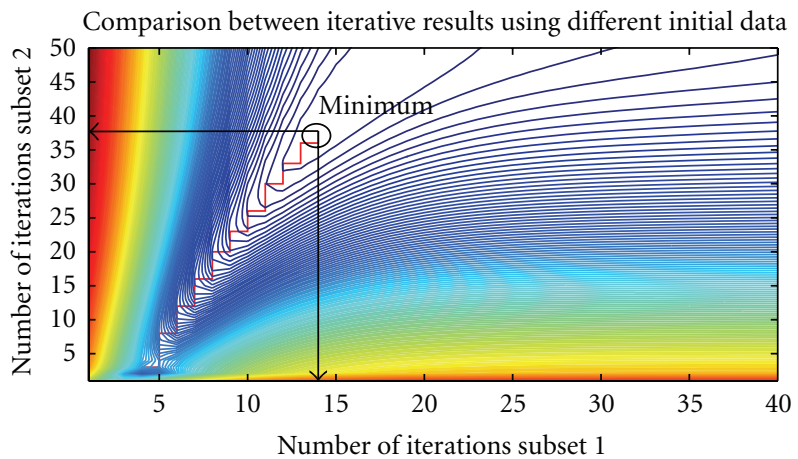

Figure 7: Gradient descent algorithm to find the optimum termination point.

exact initial data within the reliable region (see solid blue line). However, it is impossible to obtain those exact values because of the mentioned ripple errors. Then, in order to simulate the real behavior of the error, the PWS obtained from the truncated near-field acquisition is employed. Using this PWS and the spectral reliable region defined by geometrical optics and specified in Table 1, the error curve is as the red dashed line shows. In order to obtain a better convergence, the use of reliable regions smaller than that one defined by geometrical optics is considered. These new regions are determined by redefining the maximum validity angles, as indicated in Table 3 in which a weighting factor $(C<1)$ is introduced. The error variation for $C=0,8$, $C=0,6$, and $C=0,4$ is presented in Figure 5, such that when the value of $C$ is reduced, the minimum error that is achieved with this method is smaller, and the number of iterations that are required to obtain that minimum is larger. However, when that region is smaller than a certain size, the minimum error starts to increase because in addition to removing ripple errors, also useful information is removed. From practical results, we deduce that, in a general case, a value of $C$ between 0.35 and 0.85 will usually give a low minimum error. In Figure 6, the error as a function of the number 


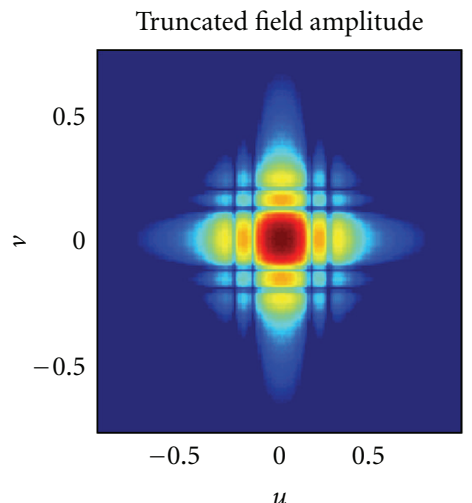

(a)

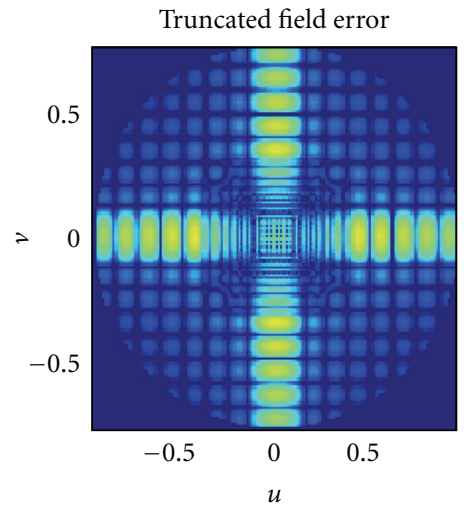

(c)

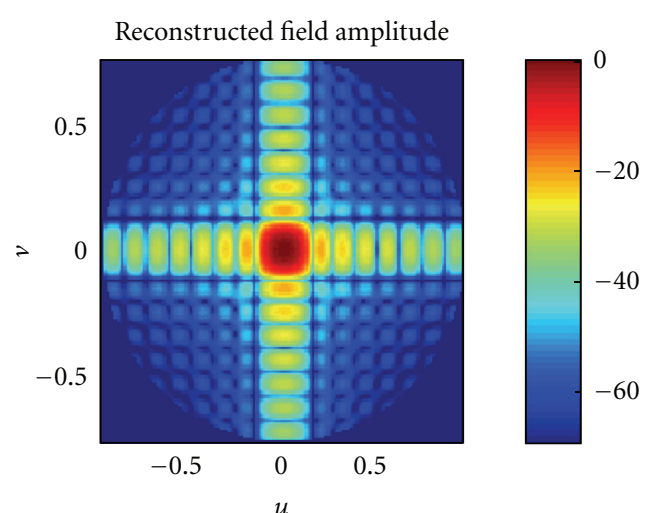

(b)

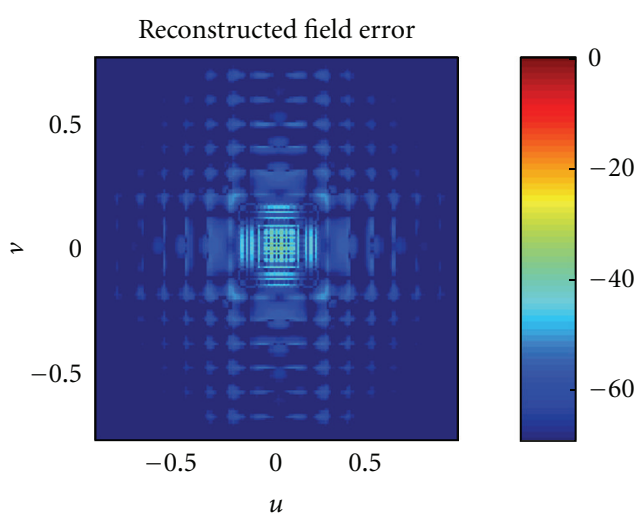

(d)

FIgURE 8: Far-field pattern and truncation error in $\mathrm{dB}$ before and after applying the iterative method for the Model I.

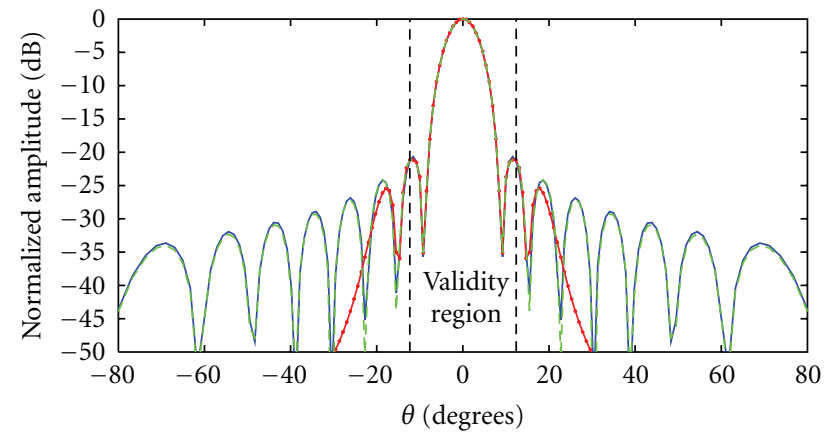

- Reference pattern $\because$ Truncated pattern

--- Reconstructed pattern

FIGURE 9: Comparison between the truncated, reconstructed and reference far-field patterns for the Model I in the $\phi=0^{\circ}$ cut.

of iterations and the parameter $C$ is depicted for the Model I. As observed, very good results are obtained in the mentioned range of $C$, with an error outside of the reliable region lower than $5 \%$.

5.3. Algorithm to Determine the Proper Termination of the Iterations. Because of the impossibility of determining the

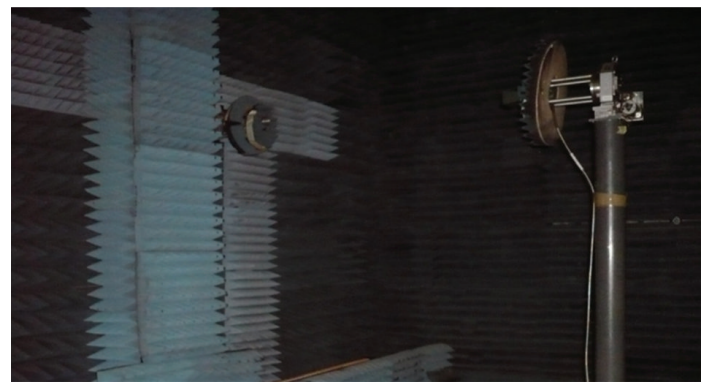

FIGURE 10: Measurement of a rectangular-horn antenna in a planar near-field range.

exact values of the PWS within the reliable region, the error in the iterative method does not decrease monotonically with the iteration number. Nevertheless, as observed in Figure 5, there is always a minimum that can provide an accurate result, and therefore, it can be used as termination point in the iterative part of the method. An algorithm to find that minimum was proposed in [17], and it is based on the following principle: if we use different initial truncated surfaces, the initial error within the reliable region will also be different, and the error in the iterative procedure will vary 


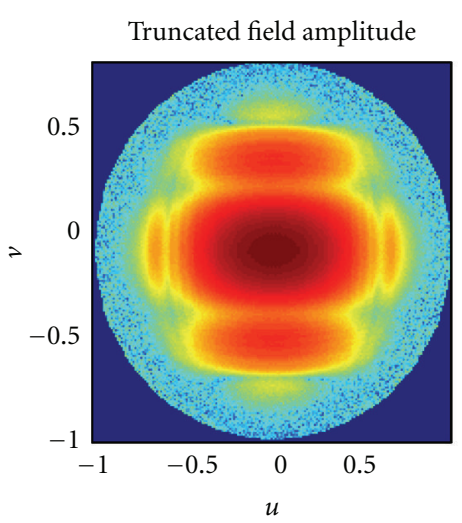

(a)

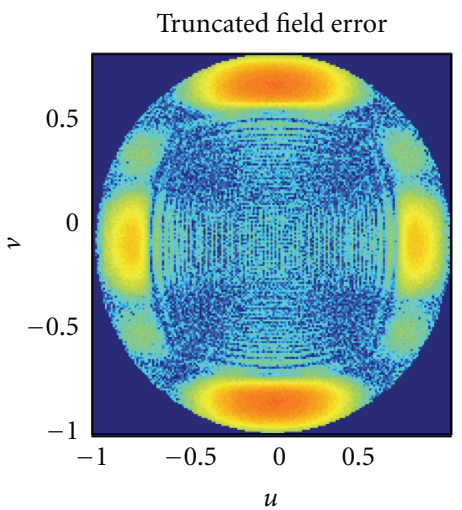

(c)

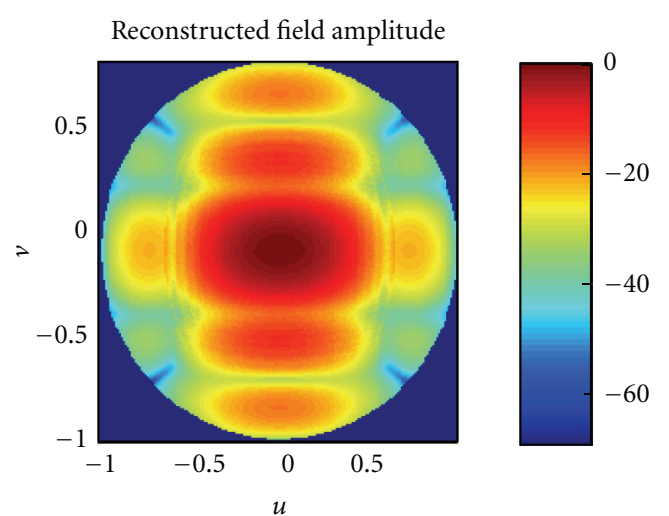

(b)

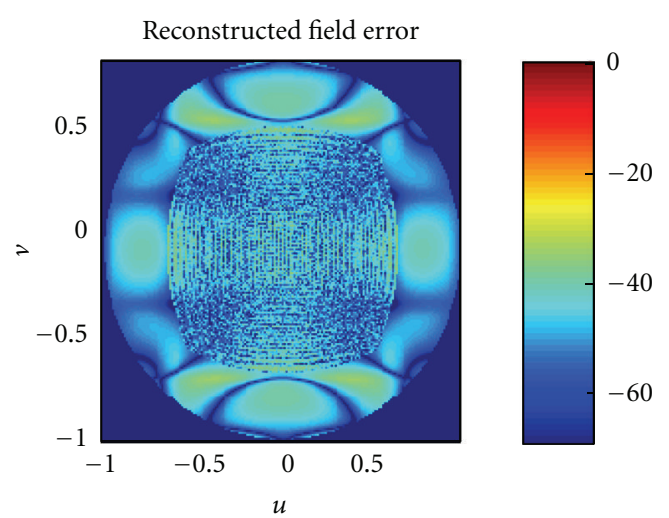

(d)

FIGURE 11: Far-field pattern and truncation error in $\mathrm{dB}$ before and after applying the iterative method for the rectangular-horn measured in PNF.

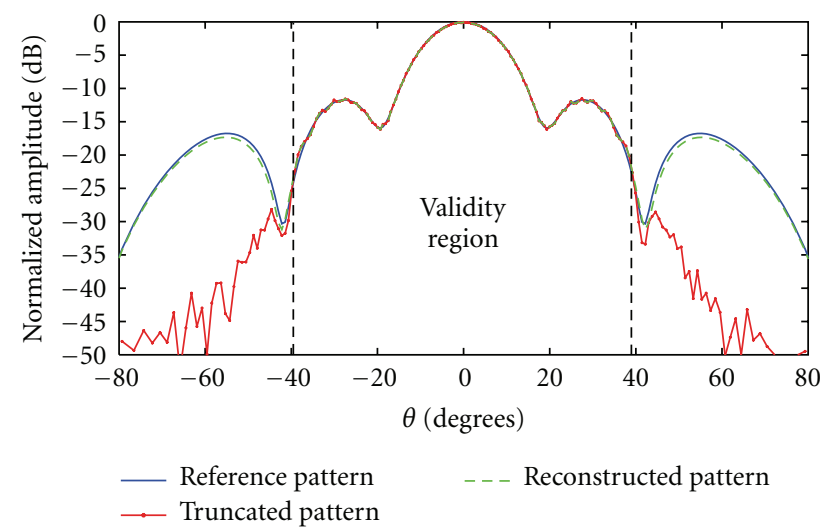

Figure 12: Comparison between the truncated, reconstructed and reference far-field patterns for the rectangular horn in the $\phi=90^{\circ}$ cut.

in a different way for the same value of $C$. Therefore, when comparing the iterative results that were obtained in both cases, we will have a minimum when both results have the minimum error. This algorithm does not require additional measurements because two different subsets of the measured near-field data can be used as inputs in the iterative method.
The iterative results obtained in both cases are stored and pairwise-compared to determine the optimum termination point for the two cases when the result of the comparison is minimum. The comparisons are carried out as follows:

$$
\mu_{i j}=\sum\left|E_{i}{ }^{1}(\theta, \phi)-E_{j}^{2}(\theta, \phi)\right|^{2}
$$

where $E_{i}{ }^{1}(\theta, \phi)$ and $E_{j}{ }^{2}(\theta, \phi)$ are the reconstructed field in the $i$ th iteration using the first data subset and the reconstructed field in the $j$ th iteration using the second data subset, respectively.

The main drawback of this approach is that many iterations and comparisons are required because we do not know where the minimum is a priori. To solve this problem, we propose the use of the Gradient Descent algorithm, which is an optimization algorithm to find the minimum. As observed in Figure 7, with this new algorithm, when we move in one direction, we perform a new iteration with one of the two data subsets and obtain a new far-field pattern estimation that is used to perform a new comparison. If the value of this new comparison is larger than the previous one, we change the direction of movement (the iterations are performed using the other data subset). If not, the algorithm performs another iteration using the same data subset. Finally, when the minimum is reached, the algorithm stops.Using 


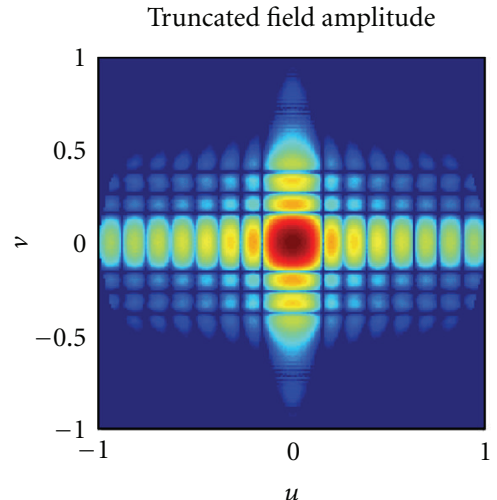

(a)

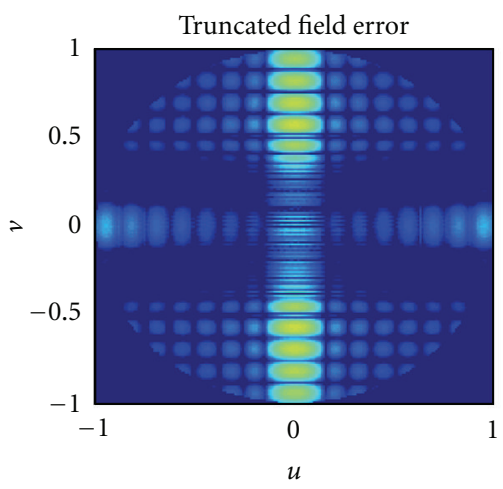

(c)
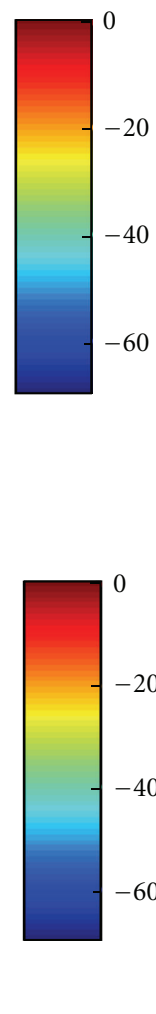

(b)

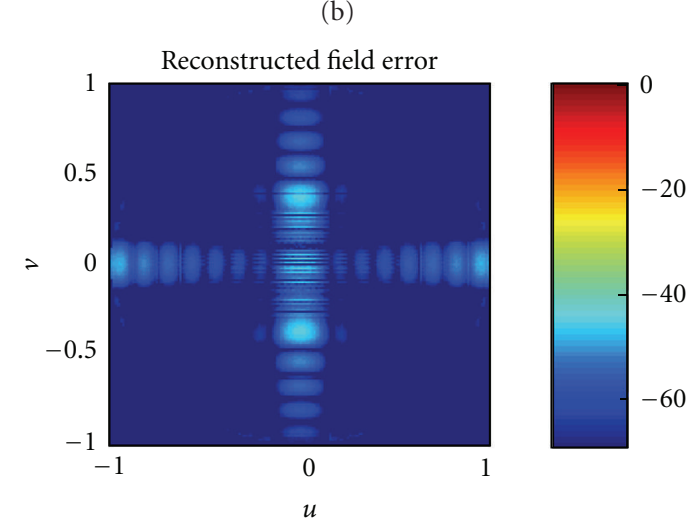

(d)

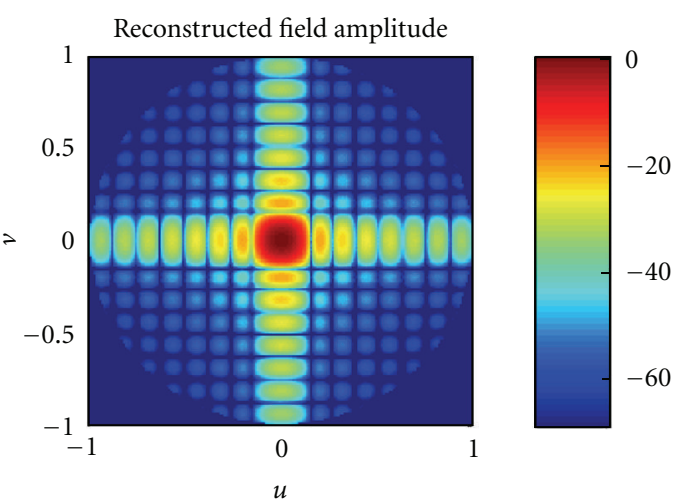

FIGURE 13: Far-field pattern and truncation error in $\mathrm{dB}$ before and after applying the iterative method for the Model II.

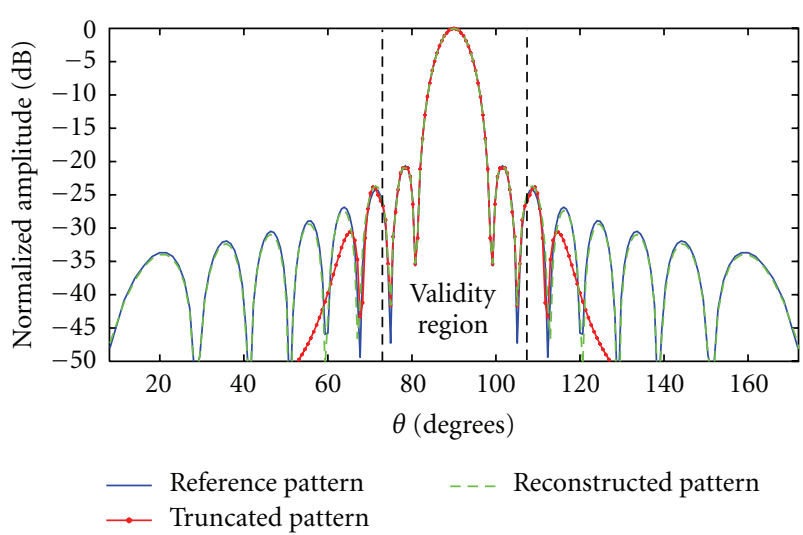

FIGURE 14: Comparison between the truncated, reconstructed and reference far-field pattern for the Model II in the $\phi=90^{\circ}$ cut.

this algorithm, both the number of iterations and comparisons may be drastically reduced, thereby requiring less computational time to obtain the minimum.

\section{Numerical Results}

To verify the accuracy of the proposed method, several examples are analyzed. The objective is to validate the method in all of the cases that are described in Figure 1. This valid- 


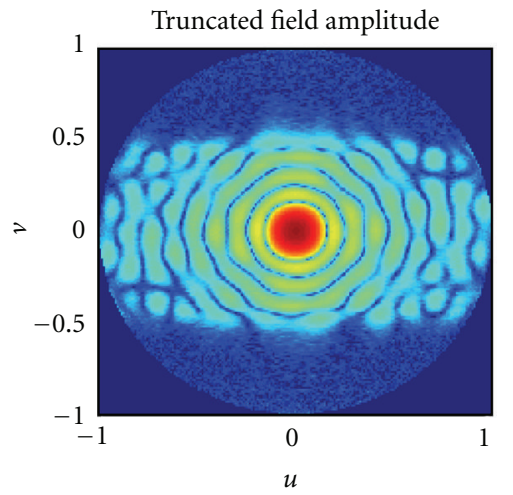

(a)

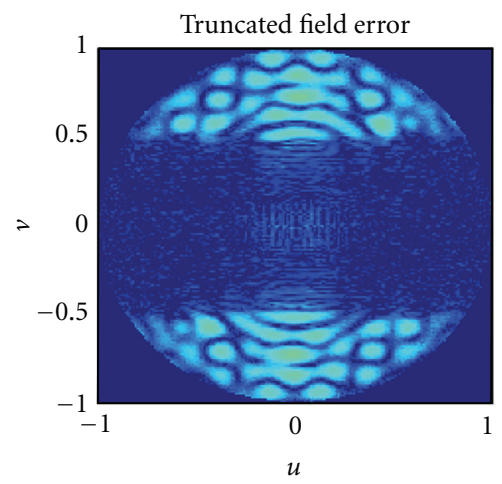

(c)

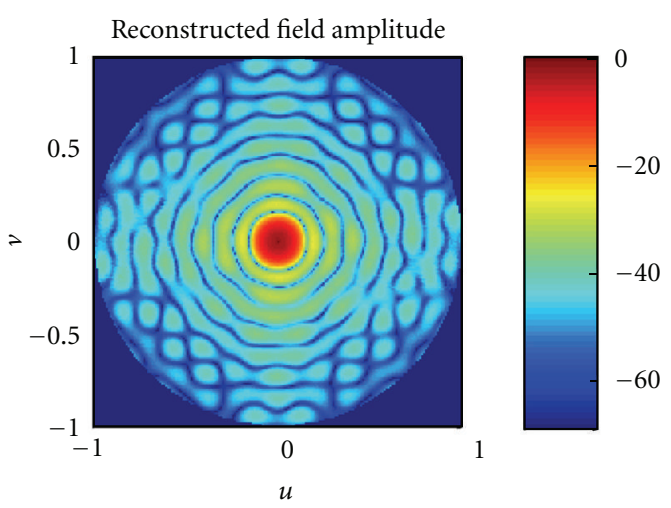

(b)

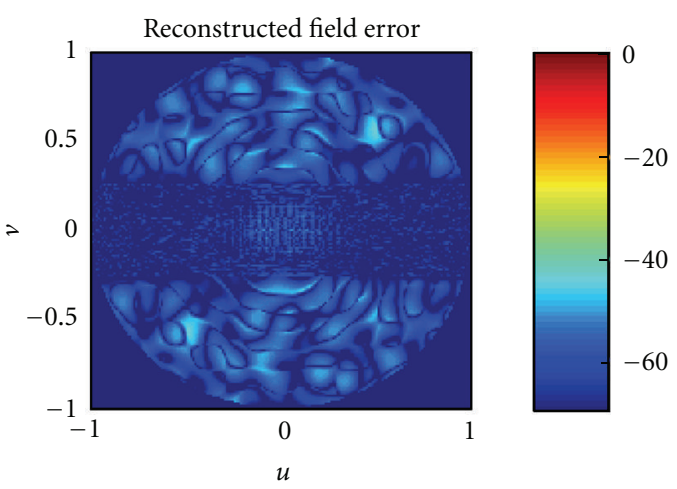

(d)

FIGURE 16: Far-field pattern and error in $\mathrm{dB}$ before and after applying the iterative method for the reflector measured in CNF.

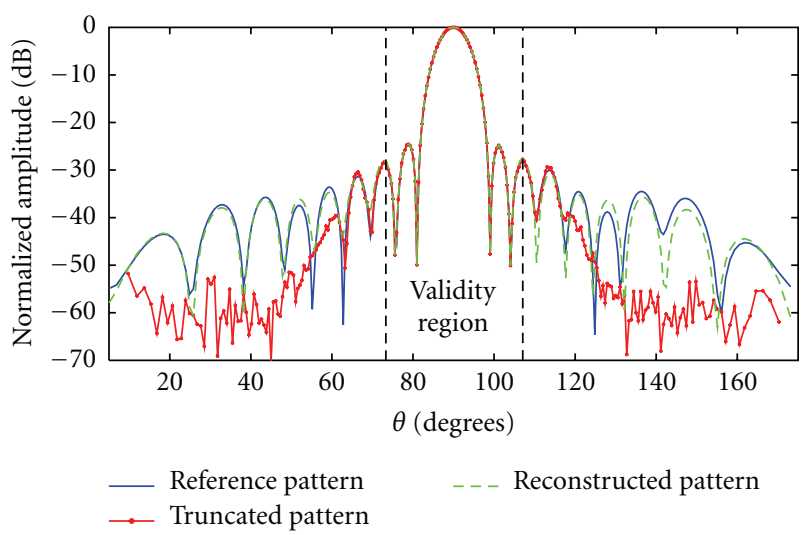

Figure 17: Comparison among the truncated, reconstructed, and reference far-field patterns for the reflector in the $\phi=90^{\circ}$ cut.

of $1.5 \mathrm{~m}$ side. The results of the reconstruction are depicted in Figure 8 in which both the far-field and the truncation error before and after the application of the method are presented. As it is apparent, the proposed procedure provides a great reduction of the truncation error and retrieves the far-field pattern in the forward hemisphere with good accuracy. In this particular case, the error defined in (18) is reduced from $62.3 \%$ to $1.2 \%$. The improvement achieved with the method is observed better in Figure 9, where a comparison among the truncated, reconstructed and reference far-field patterns for the $\phi=0^{\circ}$ cut is shown.

In the second validation, measured data were employed as input information to the proposed method. The measurement was carried out at $11 \mathrm{GHz}$ using the PNF range in the anechoic chamber at the Technical University of Madrid (UPM). The probe and the AUT were selected to be a corrugated conical-horn antenna and a rectangular-horn antenna, respectively, and they were separated from each other by $1.3 \mathrm{~m}$ (see Figure 10). When both antennas were mounted on their respective positioners, a measurement over a $2.7 \mathrm{~m} \times$ $2.7 \mathrm{~m}$ acquisition plane was recorded. The same AUT was previously measured in a SNF range in order to obtain a reference pattern for comparison with the results obtained with the presented method. Figure 11 shows a comparison between the truncated and reconstructed far-field patterns and the error before and after applying the method. As in the previous example, a great improvement of the accuracy outside of the reliable region is achieved, which reduces the error from $79.3 \%$ to $7.1 \%$. Another comparison is presented in Figure 12.

6.2. Cylindrical Near-Field Measurement. As commented before, when measuring an antenna over a cylindrical surface, an unavoidable truncation error appears in the far-field pattern because of the finite size of that surface. Therefore, 

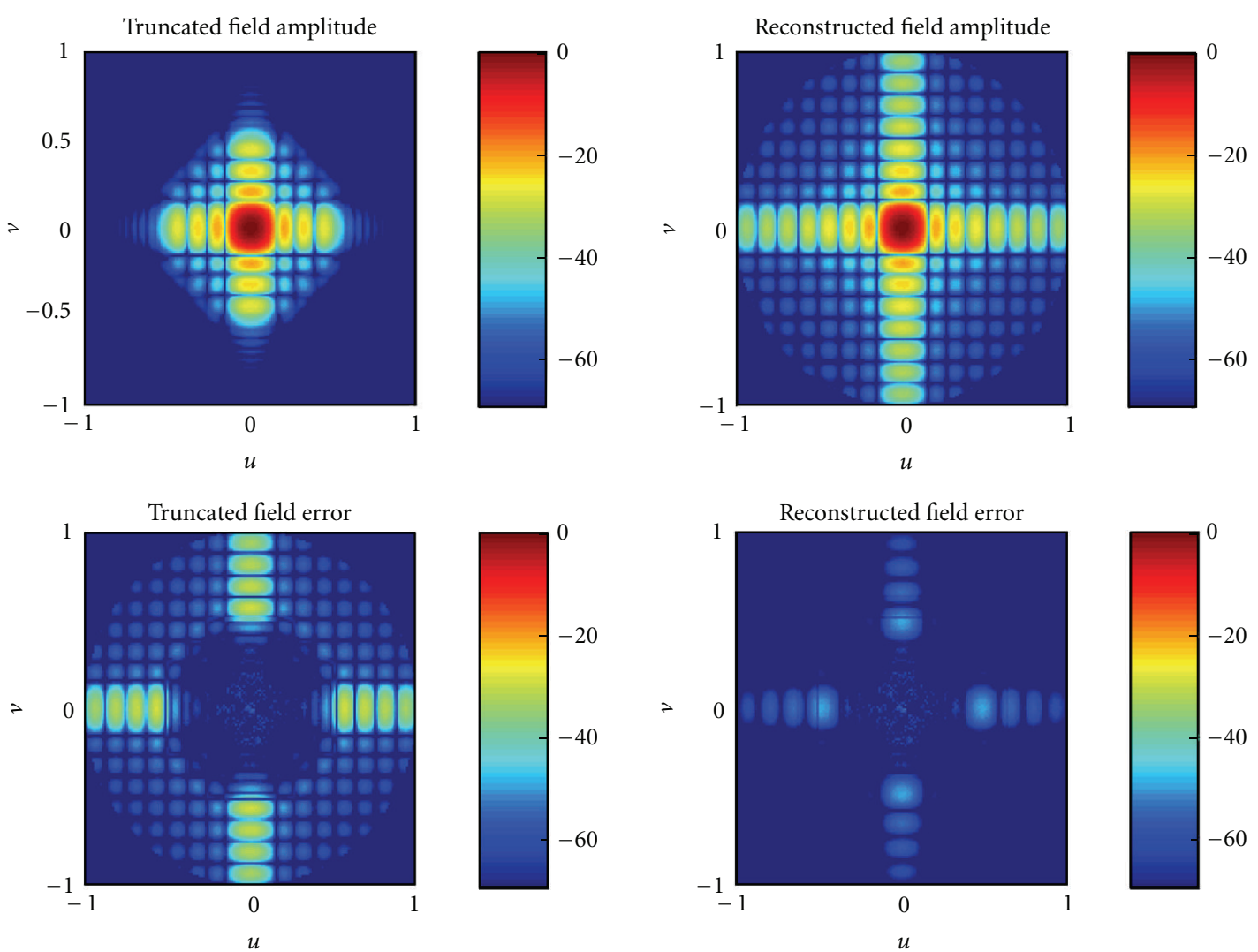

(a)
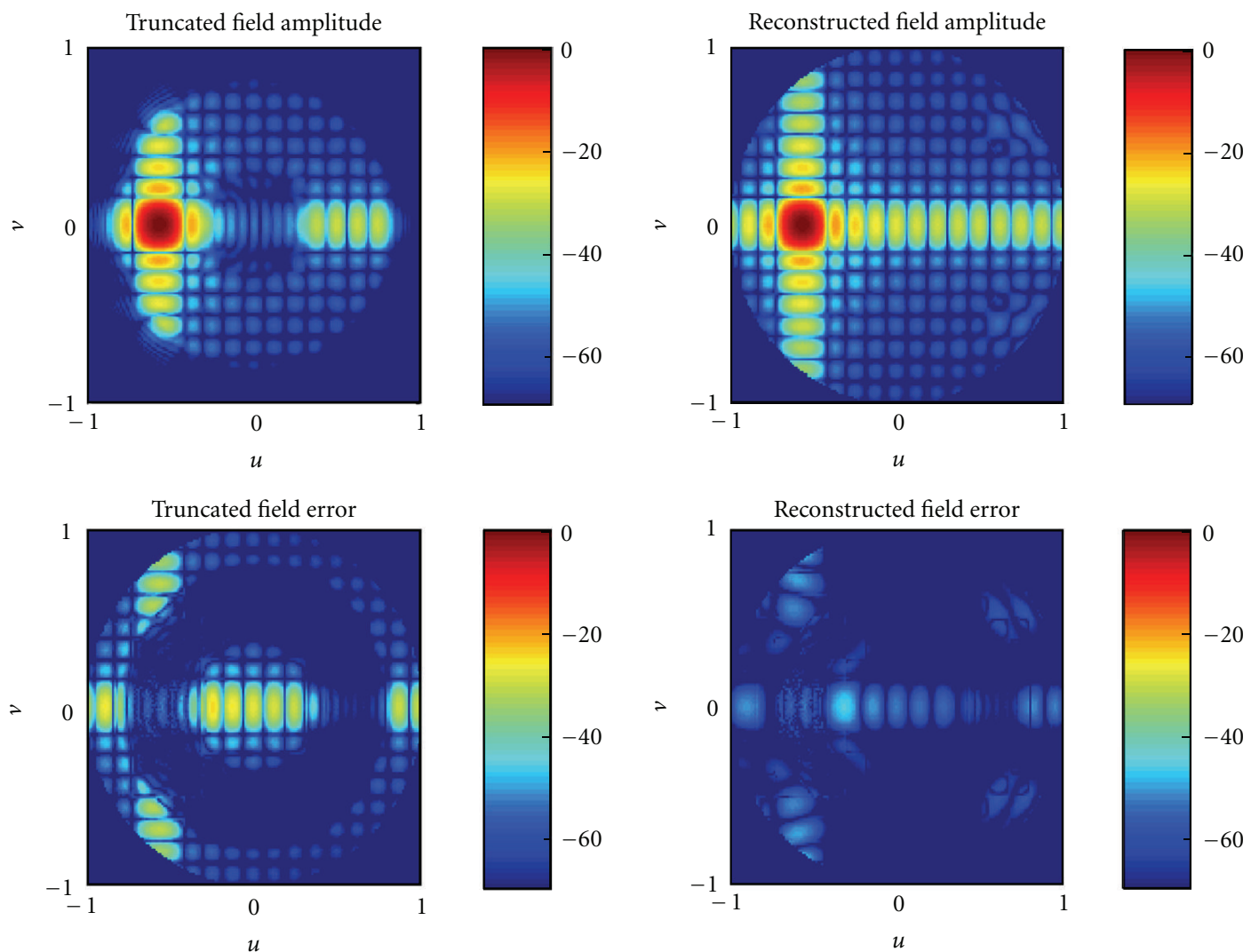

(b)

Figure 18: Continued. 

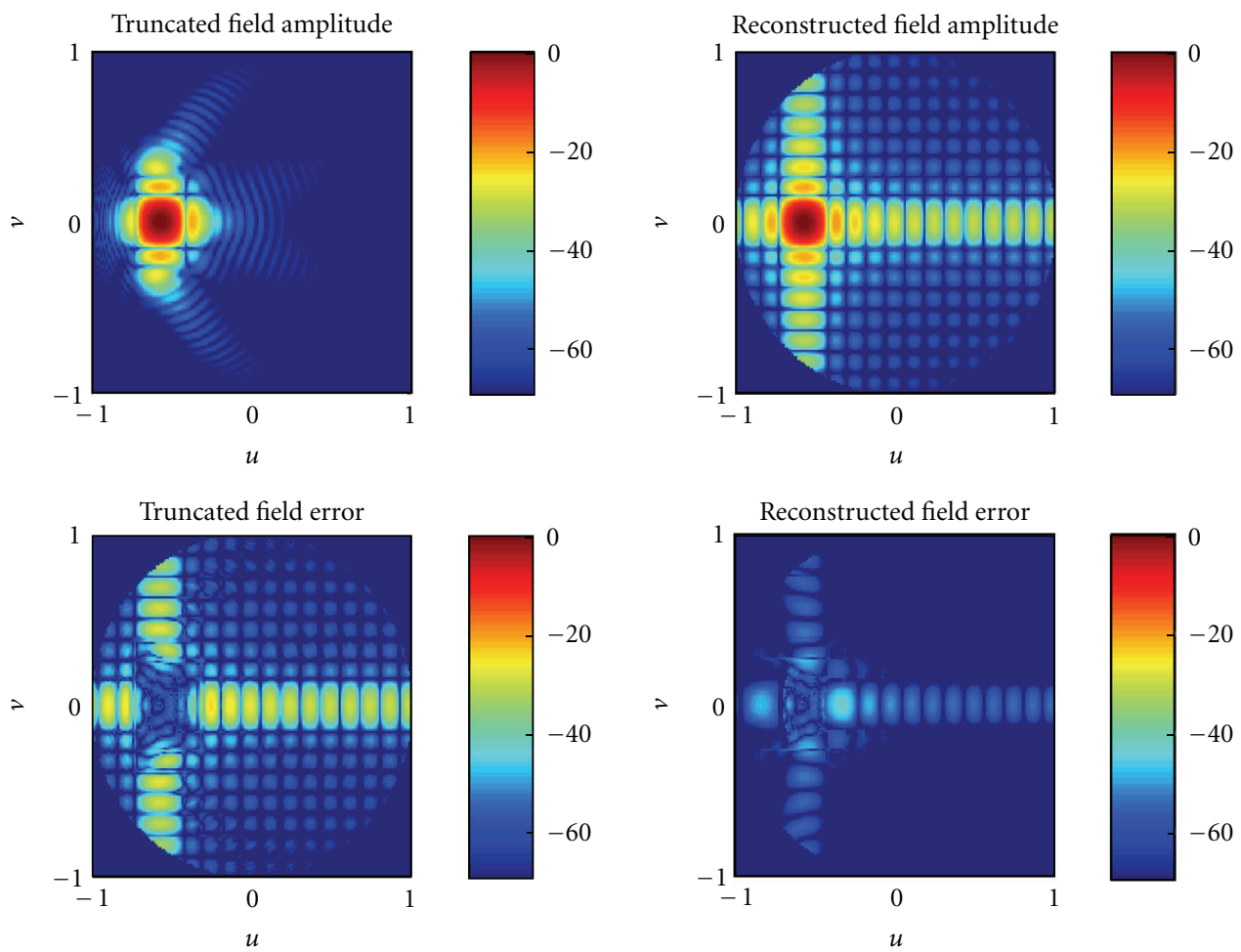

(c)
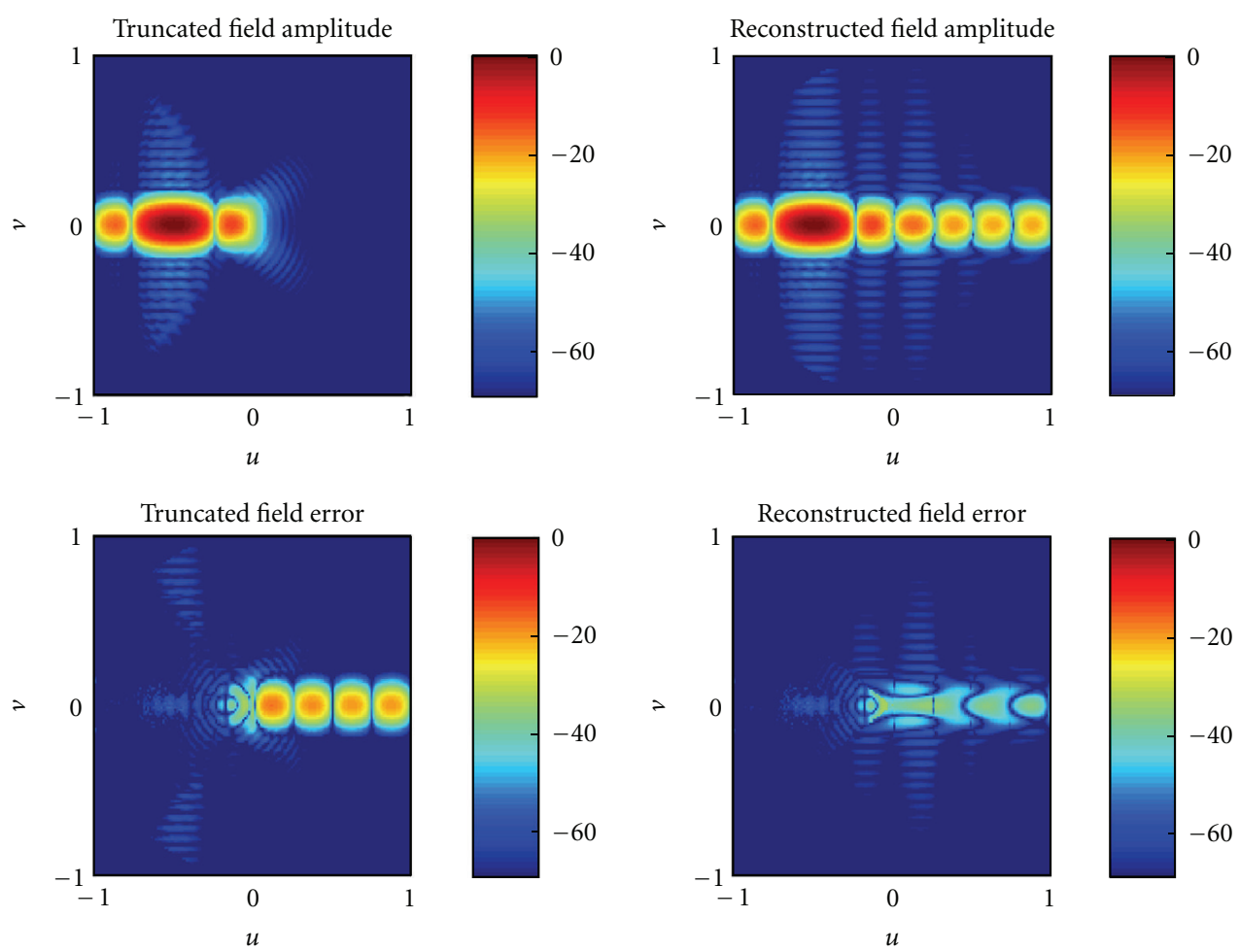

(d)

FIGURE 18: Far-field pattern and error in dB before and after applying the iterative method for the Models III (a), IV (b), V (c), and VI (d). 


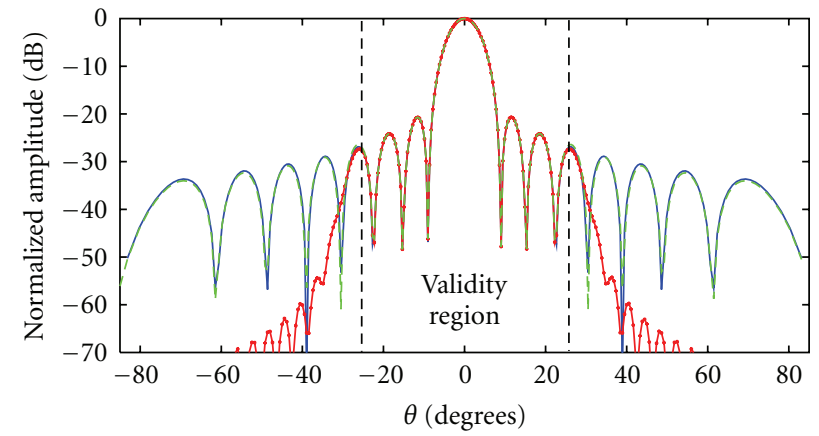

(a)

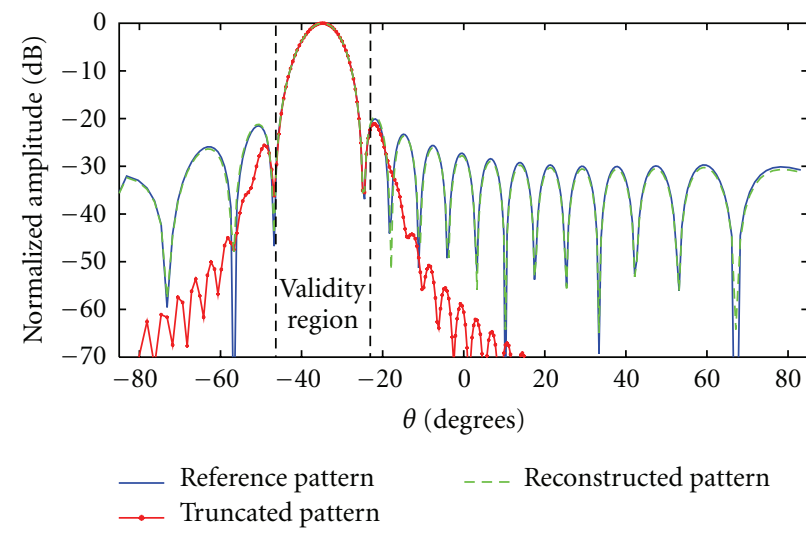

(c)

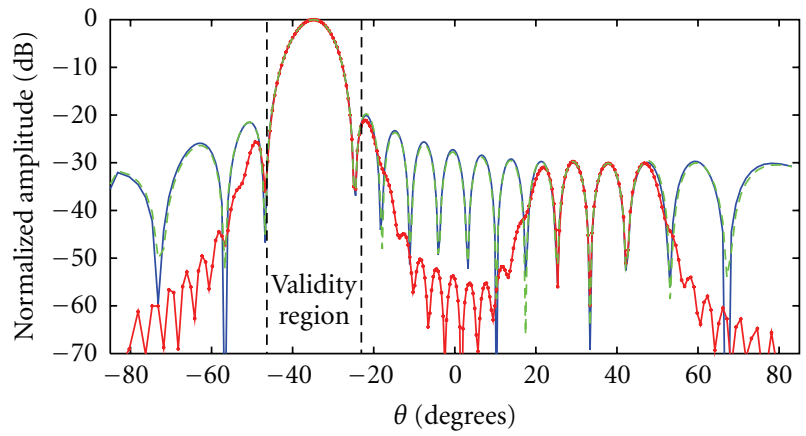

(b)

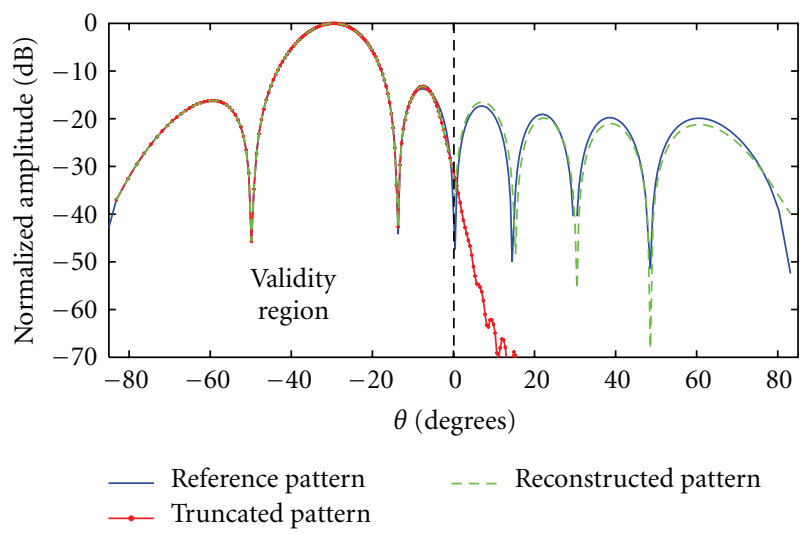

(d)

FIGURE 19: Comparison between the truncated, reconstructed and reference far-field patterns for the Models III (a), IV (b), V (c), and VI (d) in the $\phi=0^{\circ}$ cut.

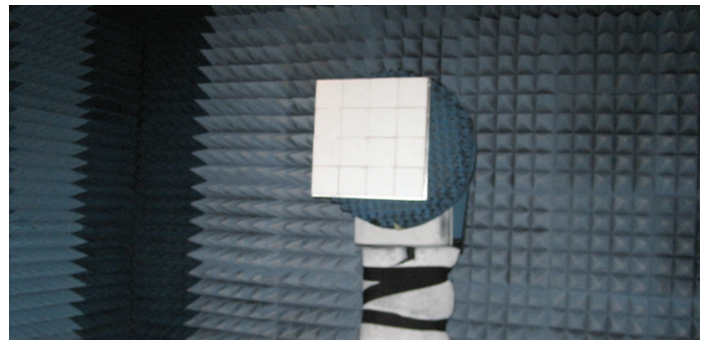

Figure 20: Measurement of an X-band array antenna in spherical near-field.

in this second part of the numerical results, cylindrical nearfield data are employed as input to the iterative method in order to demonstrate its effectiveness in this type of measurement setup.

Logically, because the method uses information about the PWS, only the truncation error in the forward hemisphere may be suppressed. The validation is carried out, as in the previous case, by employing both simulated and measured data. First, the simulated Model II that is described in Table 2 is used. According to the geometrical optics, in this first example, only the far-field pattern in the spectral region defined by $|v|<\sin \left(24.7^{\circ}\right)$ can be considered reliable. However, after the application of the method, it is possible to retrieve the pattern in the whole forward hemisphere with good accuracy, as observed in Figure 13. As in the previous examples, one far-field cut comparison is depicted in Figure 14.

The method was also validated with measured nearfield data. The measurement was performed in the CNF range at the UPM. For the experiment, the probe and the AUT consisted of a corrugated conical-horn antenna and a Ku-band reflector with a $40 \mathrm{~cm}$ diameter (see Figure 15), respectively. The data were acquired over a cylinder with a height of $2.7 \mathrm{~m}$ and a radius of $2.3 \mathrm{~m}$ and with a spatial sampling equal to $0.5 \lambda$ in the vertical direction and $2.5^{\circ}$ in the azimuth. As in the PNF case, the AUT was also measured in a whole sphere in order to obtain a reference pattern. From inspection of Figure 16, it is evident that the truncation error is greatly suppressed, reducing the error expressed in (18) from $58.2 \%$ to $8.9 \%$. A comparison depicted in Figure 17 shows the reconstructed far-field pattern in the vertical plane versus the truncated and reference far-field pattern.

6.3. Spherical Near-Field Measurement. Finally, the capability of the proposed method to reduce truncation errors in partial SNF measurements is demonstrated. Unlike PNF and CNF measurements, in which the shape of the reliable region is always the same, data can be acquired over different truncated spheres in the spherical case, defining different reliable 


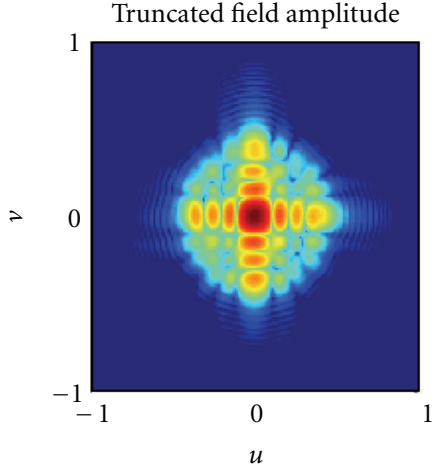

(a)

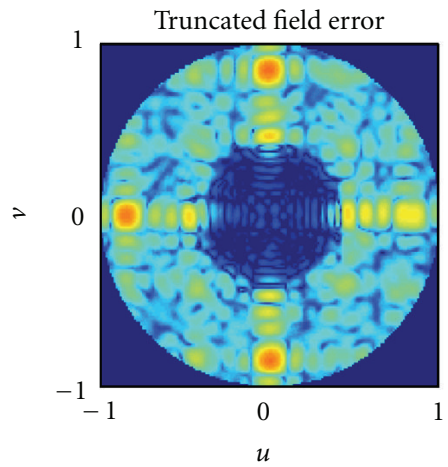

(c)
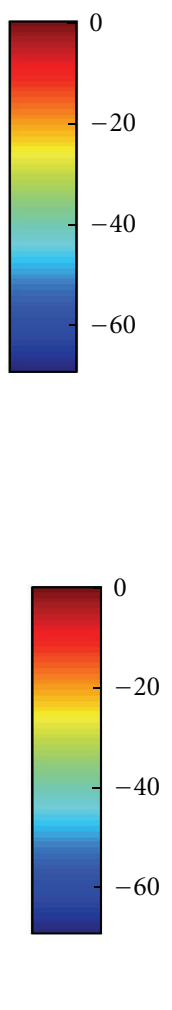

$>$

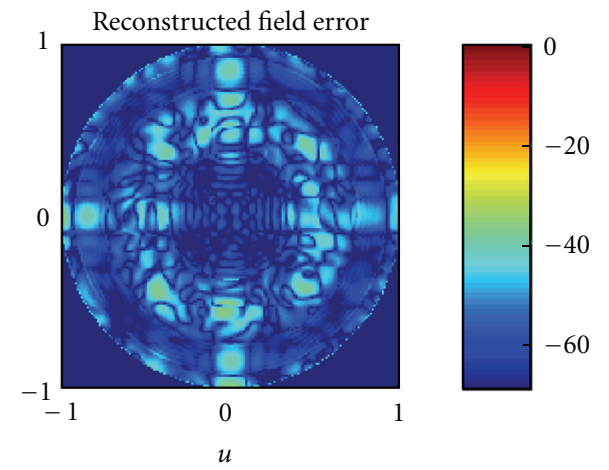

(d)

FIGURE 21: Far-field pattern and error in $\mathrm{dB}$ before and after applying the iterative method for the array antenna measured in SNF with polar truncation.

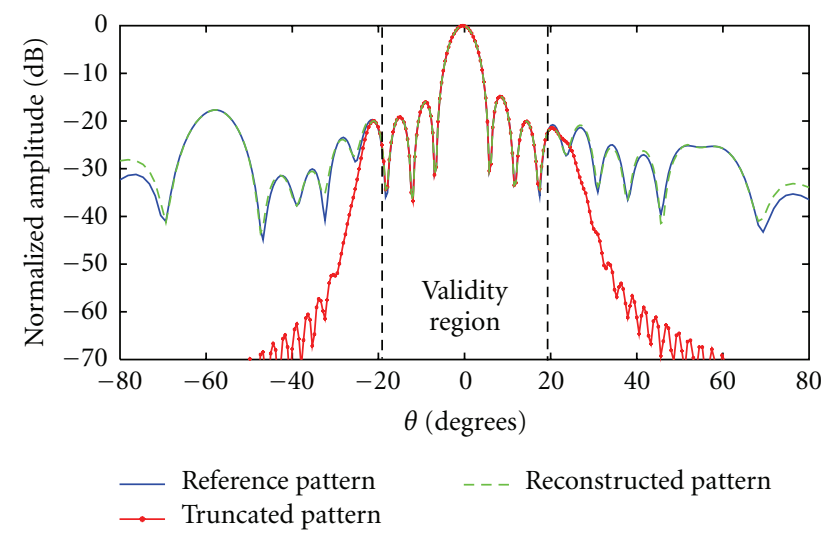

Figure 22: Comparison between the truncated, reconstructed and reference far-field pattern for the array in the $\phi=0^{\circ}$ cut.

regions. In this work, four types of truncation in the SNF measurements are considered (see Figure 2). The last four simulated models presented in Section 4 are used to analyze the effectiveness of the method in each of these truncations. All of the results are shown in Figures 18 and 19, and they contain the far-field pattern and the error before and after applying the method, as well as a comparison among the reconstructed, truncated, and reference patterns in one plane, as in the previous examples.

The proposed iterative method was also applied to measured spherical near-field data. The acquisition was performed in the SNF range at the UPM. The AUT was formed by a square array of 256 printed elements covering a large bandwidth in the X-band, and its dimensions were $40 \mathrm{~cm} \times$ $40 \mathrm{~cm}$. The array was divided into 16 square subarrays of 4 elements $\times 4$ elements (see Figure 20). Data were taken in a whole sphere with a spatial sampling equal to $2^{\circ}$ both in azimuth and elevation. Because the AUT is steering at broadside, the most appropriate truncation is a polar truncation. Therefore, only measured data from $\theta=0^{\circ}$ to $\theta=20^{\circ}$ were used as input for the method. After the application of the iterative procedure and comparison with the reference pattern from the whole measurement, the results in Figures 21 and 22 were obtained, in which the truncation error is greatly reduced, as in the previous examples. In this last case, the error of (18), which has been considered to be the quality factor, is reduced from $82.5 \%$ to $8.6 \%$.

\section{Conclusions}

An efficient method to reduce truncation errors when measuring an antenna in planar, cylindrical or partial spherical 
near-field setup has been proposed in this paper. The method is based on the Gerchberg-Papoulis iterative algorithm used to extrapolate band-limited functions, and it is a generalization of the approach presented in [17] for the planar case. Therefore, the proposed method can be viewed as a continuation of the work developed in [17], not only extending its applicability, but also introducing new algorithms to reduce the computational time required to remove the truncation errors. The convergence of this method has been mathematically demonstrated. Moreover, a detailed study of the spectral reliable region for each type of measurement setup and an analysis of critical aspects of the method has been performed. Finally, the method has been validated by using both simulated and measured near-field data, showing that it is possible to reduce the truncation errors effectively. It was noted that the proposed method works well for planar aperture antennas because the antenna aperture, in which the fields are assumed to be concentrated, is well defined.

\section{Acknowledgments}

This work was developed with the support of the Spanish FPU Grant for Ph.D. students and the financing of the Crocante Project (TEC2008-06736-C03-01/TEC). F. J. CanoFácila would also like to thank DTU Electrical Engineering for its support during his stay at the Technical University of Denmark.

\section{References}

[1] A. D. Yaghiian, "An overview of near-field antenna measurements," IEEE Transactions on Antennas and Propagation, vol. AP-34, no. 1, pp. 30-44, 1986.

[2] H. G. Booker and P. C. Clemmow, "The concept of an angular spectrum of plane waves, and its relation to that of polar diagram and aperture distribution," Proceedings of the IEEE: Radio and Communication Engineering, vol. 97, no. 45, pp. 1117, 1950.

[3] J. E. Hansen, Ed., Spherical Near-field Antenna Measurements, Peter Peregrinus, London, UK, 1988.

[4] W. M. Leach and D. T. Paris, "Probe compensated near-field measurements on a cylinder," IEEE Transactions on Antennas and Propagation, vol. AP-21, no. 4, pp. 435-445, 1973.

[5] P. R. Rousseau, "The planar near-field measurement of a broad beam antenna using a synthetic subarray approach," in Proceedings of the IEEE Antennas and Propagation Society International Symposium Digest, pp. 160-163, Montreal, Canada, July 1997.

[6] T. Al-Mahdawi, "Planar near-field measurement of low directivity embedded antennas using a synthetic array probe technique," in Proceedings of the IEEE International Antennas and Propagation Symposium Digest, pp. 1311-1314, June 1998.

[7] E. B. Joy, "Windows '96 for planar near-field measurements," in Proceedings of the Antenna Measurement Techniques Association, pp. 80-85, 1996.

[8] E. B. Joy, C. A. Rose, A. H. Tonning, and EE6254 Students, "Test-zone field quality in planar near-field measurements," in Proceedings of the Antenna Measurement Techniques Association, pp. 206-210, Williamsburg, Va, USA, November 1997.
[9] P. Petre and T. K. Sarkar, "Planar near-field to far-field transformation using an equivalent magnetic current approach," IEEE Transactions on Antennas and Propagation, vol. 40, no. 11, pp. 1348-1356, 1992.

[10] S. F. Gregson, C. G. Parini, and J. McCormick, "Development of wide-angle antenna pattern measurements using a probecorrected polyplanar near-field measurement technique," vol. 152, no. 6, pp. 563-572.

[11] O. M. Bucci, G. D'Elia, and M. D. Migliore, "New strategy to reduce the truncation error in near-field/far-field transformations," Radio Science, vol. 35, no. 1, pp. 3-17, 2000.

[12] O. M. Bucci, C. Gennarelli, and C. Savarese, "Representation of electromagnetic fields over arbitrary surfaces by a finite and nonredundant number of samples," IEEE Transactions on Antennas and Propagation, vol. 46, no. 3, pp. 351-359, 1998.

[13] J. C. Bolomey, O. M. Bucci, L. Casavola, G. D’Elia, M. D. Migliore, and A. Ziyyat, "Reduction of truncation error in nearfield measurements of antennas of base-station mobile communication systems," IEEE Transactions on Antennas and Propagation, vol. 52, no. 2, pp. 593-602, 2004.

[14] F. D'Agostino, F. Ferrara, C. Gennarelli, R. Guerriero, and G. Riccio, "An effective technique for reducing the truncation error in the near-field-far-field transformation with planepolar scanning," Progress in Electromagnetics Research, vol. 73, pp. 213-238, 2007.

[15] O. M. Bucci and M. D. Migliore, "A new method for avoiding the truncation error in near-field antennas measurements," IEEE Transactions on Antennas and Propagation, vol. 54, no. 10, pp. 2940-2952, 2006.

[16] L. Infante, E. Martini, A. Peluso, and S. Maci, "The shadow boundary integral for the reduction of the truncation error in near-field to far-field transformations," in Proceedings of the IEEE Antennas and Propagation Society International Symposium, pp. 416-419, Washington, DC, USA, July 2005.

[17] E. Martini, O. Breinbjerg, and S. Maci, "Reduction of truncation errors in planar near-field aperture antenna neasurements using the Gerchberg-Papoulis algorithm," IEEE Transactions on Antennas and Propagation, vol. 56, no. 11, pp. 3485-3493, 2008.

[18] R. W. Gerchberg, "Super-resolution through error energy reduction," Optica Acta, vol. 21, no. 9, pp. 709-720, 1974.

[19] A. Papoulis, "A new algorithm in spectral analysis and bandlimited extrapolation," IEEE Transactions on Circuits and Systems, vol. 22, no. 9, pp. 735-742, 1975.

[20] M. Serhir, J. M. Geffrin, A. Litman, and P. Besnier, "Aperture antenna modeling by a finite number of elemental dipoles from spherical field measurements," IEEE Transactions on Antennas and Propagation, vol. 58, no. 4, Article ID 5398845, pp. 1260-1268, 2010.

[21] K. T. Kim, "Truncation-error reduction in 2D cylindri$\mathrm{cal} /$ spherical near-field scanning," IEEE Transactions on Antennas and Propagation, vol. 58, no. 6, pp. 2153-2158, 2010.

[22] K. T. Kim, "Truncation-error reduction in acoustic spherical near-field scanning," in Proceedings of the Antennas and Propagation Society International Symposium, (APSURSI '10), Toronto, Canada, July 2010.

[23] A. Papoulis, Signal Analysis, McGraw-Hill, New York, NY, USA, 1977.

[24] R. C. Wittmann, C. F. Stubenrauch, and M. H. Francis, "Spherical scanning measurements using truncated data sets," in Proceedings of the Antenna Measurement Techniques Association, pp. 279-283, Cleveland, Ohio, USA, November 2002. 
[25] E. Martini, S. Maci, and L. J. Foged, "Spherical near field measurements with truncated scan area," in Proceedings of the European Conference on Antennas and Propagation, pp. 34123414, Rome, Italy, April 2004.

[26] A. D. Yaghjian, "Upper bound errors in far-field antenna parameters determined from planar near-field measurements," NBS Technical Report 667, National Bureau of Standards, Boulder, Colo, USA, 1975.

[27] C. Cappellin, Antenna diagnostic for spherical near-field antenna measurements, Ph.D. thesis, Departament of Electrical Engineering, Technical University of Denmark, Lyngby, Denmark, 2007. 

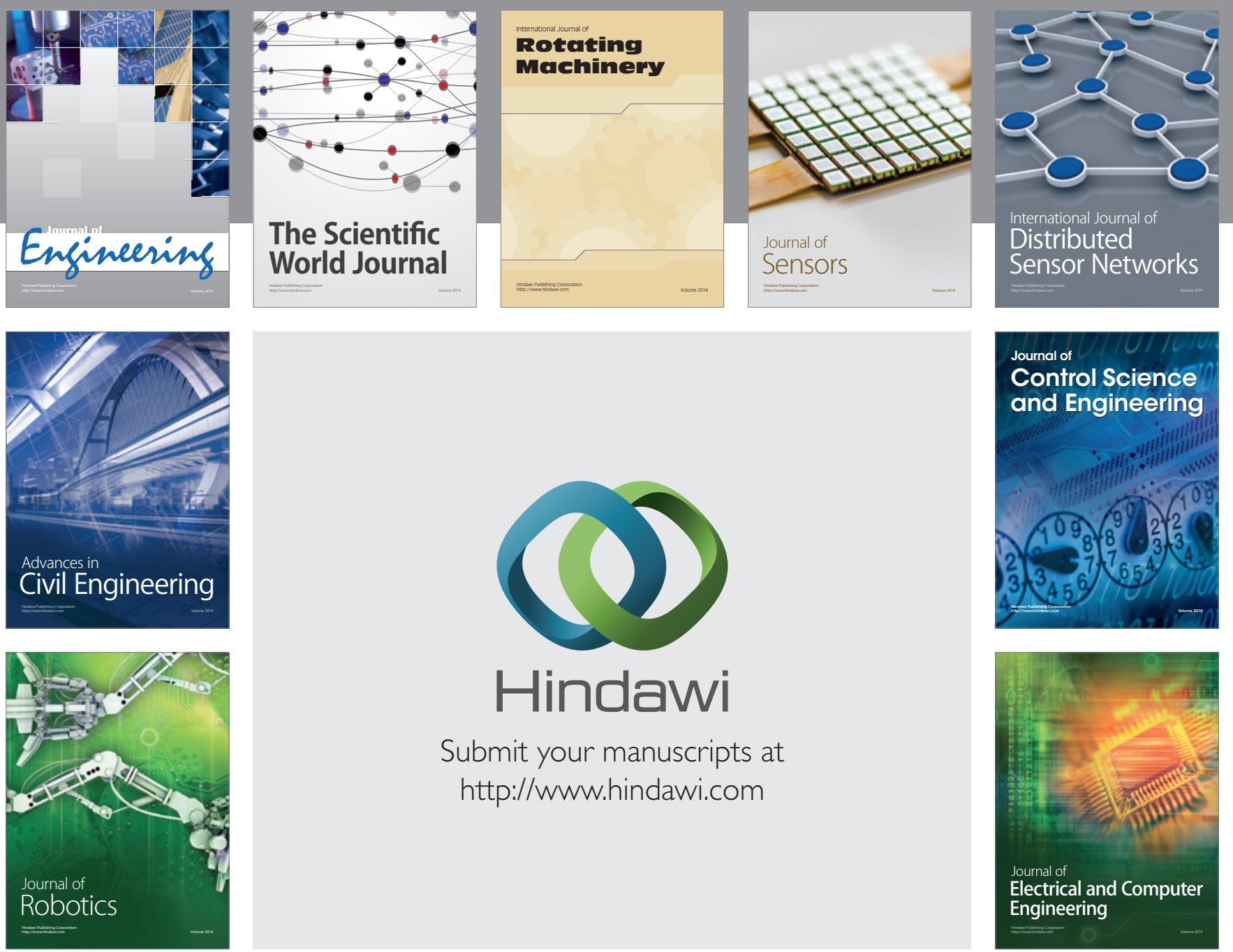

Submit your manuscripts at

http://www.hindawi.com
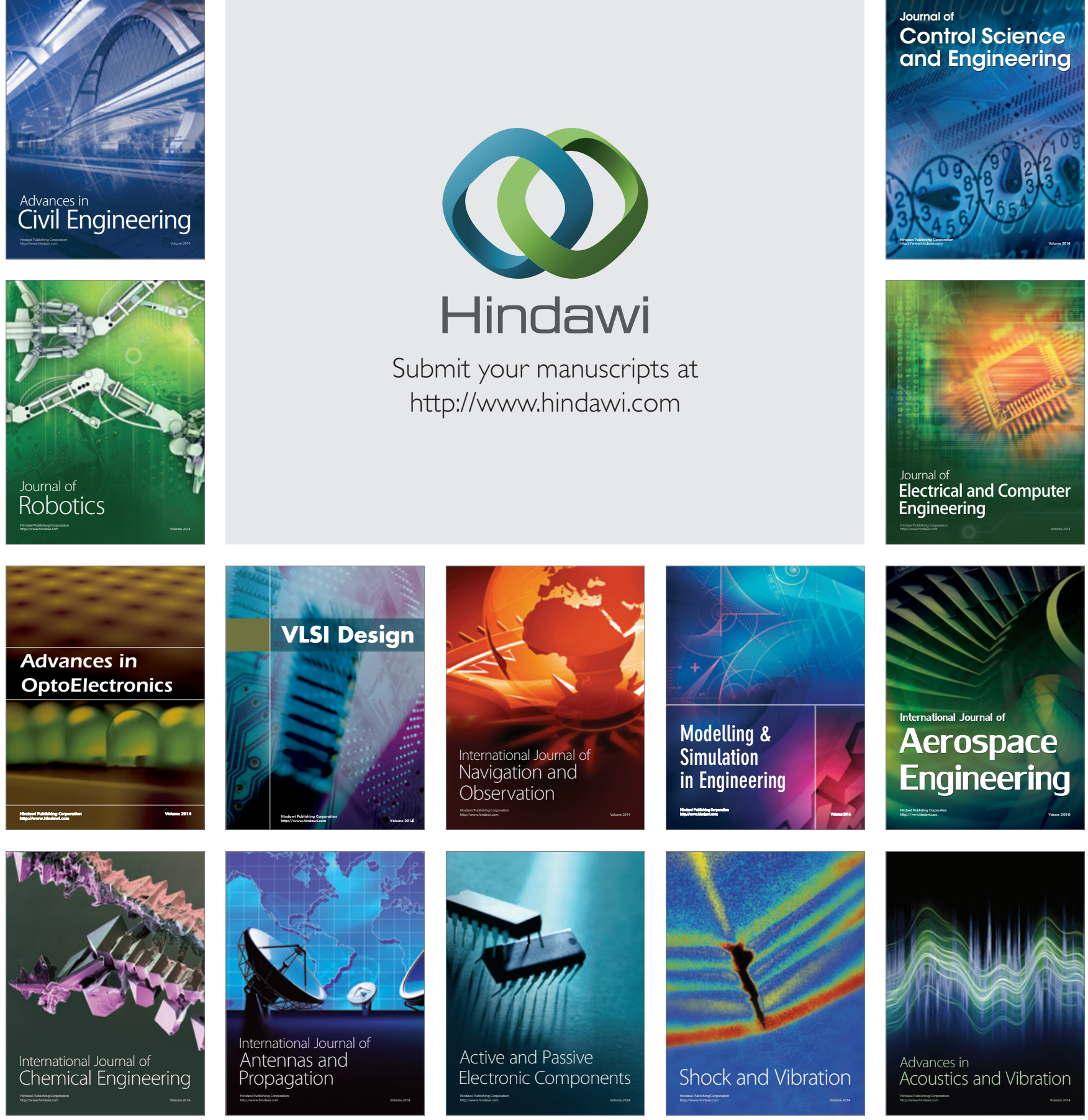